\title{
Alternative Axiomatization for Logics of Agency in a G3 Calculus
}

\author{
Sara Negri ${ }^{1}$ (D) Edi Pavlović ${ }^{2}$ (D) \\ Accepted: 30 December 2020 / Published online: 6 July 2021 \\ (c) The Author(s) 2021
}

\begin{abstract}
In a recent paper, Negri and Pavlović (Studia Logica 1-35, 2020) have formulated a decidable sequent calculus for the logic of agency, specifically for a deliberative see-to-it-that modality, or dstit. In that paper the adequacy of the system is demonstrated by showing the derivability of the axiomatization of dstit from Belnap et al. (Facing the future: agents and choices in our indeterminist world. Oxford University Press, Oxford, 2001). And while the influence of the latter book on the study of logics of agency cannot be overstated, we note that this is not the only axiomatization of that modality available. In fact, an earlier (and arguably purer) one was offered in Xu (J Philosophical Logic 27(5):505-552, 1998). In this article we fill this lacuna by proving that this alternative axiomatization is likewise readily derivable in the system of Negri and Pavlović (Studia Logica 1-35, 2020).
\end{abstract}

Keywords Logic of agency $\cdot$ Stit modalities $\cdot$ Deliberative stit $\cdot$ Sequent calculus · G3

\section{Introduction}

There has been plenty of interest in the seeing-to-it-that (stit) modalities in recent years. Notable examples of such modalities include cstit (c for Chellas), an agent-relative box-type modality as well as more involved dstit (d for deliberative) and astit (a for achievement). There is a good reason for this interest, as they can be used to unify a great variety of linguistic expressions (Belnap et al. 2001).

The treatment of stit modalities in terms of proof theory remains relatively scant, notable exceptions being (Wansing 2006, 2017; Olkhovikov and Wansing 2018, 2019), which all

This paper was written with support of the Academy of Finland research project No. 1308664.

$凶$ Edi Pavlović

Edi.Pavlovic@helsinki.fi

Sara Negri

Sara.Negri@unige.it

1 Department of Mathematics, University of Genova, Via Dodecaneso 35, 16146 Genoa, Italy

2 Department of Philosophy, History and Art Studies, University of Helsinki, P.O. Box 24, 00014 Helsinki, Finland 
involve a tableaux approach. Approaches utilizing a sequent calculus have been developed recently, in particular (using a simplification of a tableux) van Berkel and Lyon (2019); Lyon and van Berkel (2019) and Negri and Pavlović (2020) (which builds the calculus based on the semantics from Belnap et al. 2001). The system from the latter will be used in this paper, since in addition to the usual range of desirable proof-theoretic properties (like the admissibility of contraction and cut), it also offers a structural proof of multi-agent decidability and, even though it focuses on dstit, provides a uniform basis for the treatment of multiple stit modalities.

Even with those upsides, we nonetheless find that paper incomplete in one respect. It demonstrates the adequacy of the system for the treatment of dstit by showing that the axioms of dstit from Belnap et al. (2001) are derivable in it. This, however, is not the only axiomatization of that modality, with another notable formulation found in Xu (1998) (also investigated in Balbiani et al. 2008). The axiomatization there contains only the dstit operator, in contrast with the four types of modal operators occurring in Belnap et al. (2001). And while this system is clearly expressively weaker, it is also quite obviously the logic of dstit proper, as opposed to being a logic merely containing dstit.

It is the purpose of this article to demonstrate that these alternative axioms are likewise readily derivable in the system of Negri and Pavlović (2020). As such, in the proceeding we will lean heavily on the results present therein. In order to avoid repeating large swathes of that paper we will not present its proofs, but for ease of reference the results relevant for this paper will be noted in the following section. Throughout the paper we will mark any propositions, theorems or lemmas from Negri and Pavlović (2020) with an asterisk.

Here we are dealing with three systems - axiomatic ones of Belnap et al. (2001); Xu (1998) and a sequent calculus from Negri and Pavlović (2020). Given that all three are sound and complete with respect to the background semantics, one should prima facie expect the results of this paper to hold.

However, there is a conceptual priority of an existence of proof to metatheoretical resultsa system is complete because certain proofs exist (the other way around would be putting the cart before the horse), and it is standard practice in proof theory to prefer a direct proof to a roundabout one. Case in point-in Negri and Pavlović (2020) it would not be, strictly speaking, necessary to demonstrate completeness via a failed proof search after already showing that the axioms of Belnap et al. (2001) are derivable. However, the former approach, in addition to being elegant, provides the correct conceptual connection between the system and its underlying semantics. The demonstration of axioms is required since it shows, in light of the fact that axioms are the most common way of presenting a logic, that it adequately captures a logic of dstit. And insofar as it is not the only logic of dstit around, with the version from Xu (1998) arguably purer (although at the expense of brevity), the results of the present paper are required to present the full picture.

After the introduction we lay out the systems we will be comparing, and after that dedicate each section to one of the consecutive expansions of the axiomatization from Xu (1998), and give a separate subsection to the more involved proofs. Moreover, since some are outright preposterously long, their full proof will be deferred to the appendix.

\section{Preliminaries}

We begin with a note on notation-Belnap et al. (2001), Xu (1998) write "agent $\alpha$ deliberately sees to it that $A$ " as " $[\alpha: A]$." For brevity and following Negri and Pavlović (2020)— the 
decision there was likewise made to shorten the notation-we will write this as " $D^{\alpha} A$." We prefer to mark agents by numbers and thus use letters $i, j, \ldots$ instead of Greek letters for agents, except for the axioms $\mathrm{AIA}_{n}$ and $\mathrm{APC}_{k}$, where two different sets of agents are involved. The full language of dstit that we utilize is defined as

$$
A::=\perp|p| A \circ A\left|\square^{i} A\right| \mathcal{S} A|\mathcal{P} A| \mathcal{D}^{i} A
$$

where o stands for connectives, $\square^{i}$ stands for cstit (Chellas 1980), $\mathcal{S}$ for settled true and $\mathcal{P}$ for possible, the last two agent-independent (Belnap et al. 2001).

The formulas are labelled by moment/history pairs so that $m / h: A$ states that the formula $A$ holds at a moment $m$ of history $h$ ( $m \in h$ indicates history $h$ contains the moment $m$ ). In the paper the reader will also frequently encounter the choice-equivalence relation $h \sim_{m}^{i} h^{\prime}$, which can be intuitively understood as saying that at moment $m$, the agent $i$ cannot choose between the histories $h$ and $h$ (it is beyond the agent's control which one comes about). Finally, the abbreviation $\operatorname{Diff}\left(i_{1}, \ldots, i_{k}\right)$ stands for distinctness of a finite number $k$ of agents, i.e. inequalities $i_{j} \neq i_{n}$ where $1 \leq j<n \leq k$.

\subsection{The Alternative Axiomatization}

The axiom systems for dstit presented in Xu (1998) are as follows, adjusted for the notation of this paper. Each subsequent system extends the previous ones.

\section{Logic $\mathbf{L}_{0}$ :}

A0 Truth functional tautologies

$\mathbf{A 1} \mathcal{D}^{i} A \supset A$

A2 $\mathcal{D}^{i} A \supset \mathcal{D}^{i} \mathcal{D}^{i} A$

A3 $\mathcal{D}^{i} A \supset \mathcal{D}^{i} \neg \mathcal{D}^{i} \neg \mathcal{D}^{i} A$

A4 $\left(\mathcal{D}^{i} A \& \mathcal{D}^{i}(B \& C)\right) \supset \mathcal{D}^{i}(A \& C)$

A5 $\left(\mathcal{D}^{i} A \& \neg \mathcal{D}^{i} B\right) \supset \mathcal{D}^{i}\left(A \& \neg \mathcal{D}^{i} B\right)$

A6 $\mathcal{D}^{i}\left(\neg \mathcal{D}^{i} A \&\left(B \& \neg \mathcal{D}^{i} B\right)\right) \supset \subset \mathcal{D}^{i} \neg \mathcal{D}^{i}\left(A \&\left(B \& \neg \mathcal{D}^{i} B\right)\right)$.

$\operatorname{Logic} \mathbf{L}_{1}$ :

A7 $i=i$

$\mathbf{A 8} i=j \supset(A \supset A(i / j))$

A9 $\mathcal{D}^{i} A \supset \subset\left(\mathcal{D}^{i}(A \& j=k) \vee \mathcal{D}^{i}(A \& j \neq k)\right)$

$\mathbf{A 1 0}\left(\mathcal{D}^{i} A \& \mathcal{D}^{k}\left(B \&\left(C \& \neg \mathcal{D}^{k} C\right)\right)\right) \supset \mathcal{D}^{i}\left(A \&\left(C \& \neg \mathcal{D}^{i} C\right)\right)$

$\mathbf{A 1 1}\left(\mathcal{D}^{i} A \& \neg \mathcal{D}^{i}\left(A \& \neg \mathcal{D}^{k} B\right)\right) \supset\left(\mathcal{D}^{k} B \vee \mathcal{D}^{k} \neg \mathcal{D}^{k} B\right)$

$\mathbf{A 1 2} \mathcal{D}^{i} \neg \mathcal{D}^{k} B \supset i=k$

$\mathbf{A I A}_{n} \mathcal{D}^{a} A \&\left(\neg \mathcal{D}^{a}\left(A \& \neg \mathcal{D}^{b_{1}} B_{1}\right) \& \ldots \& \neg \mathcal{D}^{a}\left(A \& \neg \mathcal{D}^{b_{n}} B_{n}\right)\right) \& \operatorname{Diff}\left(b_{1}, \ldots, b_{n}\right) \supset$ $\neg \mathcal{D}^{a}\left(A \& \neg\left(\mathcal{D}^{b_{1}} B_{1} \& \ldots \& \mathcal{D}^{b_{n}} B_{n}\right)\right)$

where $\operatorname{Diff}\left(b_{1}, \ldots, b_{n}\right)$ indicates pairwise inequality ranging over all pairs of non-identical agents.

Logic $\mathbf{L}_{k}$ : Each subsequent logic $\mathrm{L}_{k}$ is determined by $k$ in $\mathrm{APC}_{k}$.

$\mathbf{A P C}_{k} \neg \mathcal{D}^{a}\left(A \& \neg\left(\mathcal{D}^{b} B_{1} \& \neg B_{2} \& \ldots \& \neg B_{k}\right)\right) \& \ldots \& \neg \mathcal{D}^{a}\left(A \& \neg\left(\mathcal{D}^{b} B_{k-1} \& \neg B_{k}\right)\right) \&$ $\neg \mathcal{D}^{a}\left(A \& \neg \mathcal{D}^{b} B_{k}\right) \& \mathcal{D}^{a} A \& a \neq b \supset \mathcal{D}^{b} B_{1} \vee \ldots \vee \mathcal{D}^{b} B_{k}$

Moreover, the rules used are those of modus ponens and RE:

$$
\frac{A \supset \subset B}{\mathcal{D}^{i} A \supset \subset \mathcal{D}^{i} B} R E
$$




\subsection{G3DSTIT and G3 Ldm $_{k}$}

We now present the sequent calculus to be used in this article. The sequent calculus is a G3-style calculus (Negri 2005; Negri and von Plato 1998, 2001, 2011), with the treatment of auxiliary modalities mirroring that of Negri and Sbardolini (2016), defined as in (Fig. 1).

This system will be used for demonstration of the adequacy of axioms up to and including $\mathrm{L}_{1}$. For any subsequent system we add the appropriate rule $\mathrm{APC}_{k}$ to G3DSTIT to produce the system G3Ldm $k$.

\subsubsection{Previous Results}

The results from Negri and Pavlović (2020) used in this article are:

1. First and foremost, all the axioms of dstit from Belnap et al. (2001) are derivable (Proposition* 3.1),

2. Axiom generalization, $m / h: A, \Gamma \Rightarrow \Delta, m / h: A$ for any formula $A$ (Lemma* 4.2), used here without special reference,

3. Height-preserving substitution (Proposition* 4.3),

4. (Lemma* 5.1): $h \sim_{m}^{i} h^{\prime}, m / h: \mathcal{D}^{i} A \Rightarrow m / h^{\prime}: \mathcal{D}^{i} A$,

5. Impossibility of meta-agency (Theorem* 5.2): $m / h: \mathcal{D}^{i} \mathcal{D}^{j} A \Rightarrow$ and $m / h: \mathcal{D}^{i} \neg \mathcal{D}^{j} A \Rightarrow$, when $\operatorname{Diff}(i, j)$,

6. (Proposition* 5.3): $\mathcal{D}^{i} \neg \mathcal{D}^{i} A \Leftrightarrow \mathcal{P} \mathcal{D}^{i} A \& \neg \mathcal{D}^{i} A$

7. Doing is equivalent to refraining from refraining (Proposition* 5.4): $m / h: \mathcal{D}^{i} A \Leftrightarrow m / h$ : $\mathcal{D}^{i} \neg \mathcal{D}^{i} \neg \mathcal{D}^{i} A$,

8. Finally, the system with the rule $\mathrm{APC}_{k}$ added is decidable (Theorem* 6.8), and decidable without the rule for a system with a single agent (Lemma* 6.11).

It is easy to show that the rules used in Xu (1998) are admissible-modus ponens is trivial, and RE uses a slight modification of the proof of axiom generalization for the case of $\mathcal{D}^{i}$.

\section{Logic Lo}

Since this system is formulated for only a single agent, we know by Lemma* 6.11 that all of its axioms are decidable. This has been particularly useful in the proof search for A6 (given its length, the proof of this axiom is found in the appendix). In fact, (the sequent calculus versions of) all of the following are derivable in G3DSTIT:

A0 Truth functional tautologies

$\mathbf{A 1} \mathcal{D}^{i} A \supset A$.

Proof

$$
\frac{h \sim_{m}^{i} h, m / h: \square^{i} A, m / h: A \Rightarrow m / h: A, m / h: \mathcal{S} A}{\frac{h \sim_{m}^{i} h, m / h: \square^{i} A \Rightarrow m / h: A, m / h: \mathcal{S} A}{\frac{m / h: \square^{i} A \Rightarrow m / h: A, m / h: \mathcal{S} A}{m / h: \mathcal{D}^{i} A \Rightarrow m / h: A}} L \square^{i}} \operatorname{Re}^{i} l_{\sim_{m}^{i}}
$$


Initial sequents: $\quad m / h: p, \Gamma \Rightarrow \Delta, m / h: p \quad m / h: \perp, \Gamma \Rightarrow \Delta$

Propositional rules: Standard G3.

\section{Modal rules:}

$$
\begin{gathered}
\frac{h^{\prime} \sim_{m}^{i} h, \Gamma \Rightarrow \Delta, m / h^{\prime}: A}{\Gamma \Rightarrow \Delta, m / h: \square^{i} A} R \square^{i} \\
\frac{h^{\prime} \sim_{m}^{i} h, m / h: \square^{i} A, m / h^{\prime}: A, \Gamma \Rightarrow \Delta}{h^{\prime} \sim_{m}^{i} h, m / h: \square^{i} A, \Gamma \Rightarrow \Delta} L \square^{i} \\
\frac{m / h: \square^{i} A, \Gamma \Rightarrow \Delta, m / h: \mathcal{S} A}{m / h: \mathcal{D}^{i} A, \Gamma \Rightarrow \Delta} L \mathcal{D}^{i} \\
\frac{\Gamma \Rightarrow \Delta, m / h: \square^{i} A m / h: \mathcal{S} A, \Gamma \Rightarrow \Delta}{\Gamma \Rightarrow \Delta, m / h: \mathcal{D}^{i} A} R \mathcal{D}^{i}
\end{gathered}
$$

$\frac{m \in h^{\prime}, m / h^{\prime}: A, m / h: \mathcal{S} A, \Gamma \Rightarrow \Delta}{m \in h^{\prime}, m / h: \mathcal{S} A, \Gamma \Rightarrow \Delta} L \mathcal{S} \quad \frac{m \in h^{\prime}, \Gamma \Rightarrow \Delta, m / h^{\prime}: A}{\Gamma \Rightarrow \Delta, m / h: \mathcal{S} A} R \mathcal{S}$ $\frac{m \in h^{\prime}, m / h^{\prime}: A, \Gamma \Rightarrow \Delta}{m / h: \mathcal{P} A, \Gamma \Rightarrow \Delta} L \mathcal{P} \quad \frac{m \in h^{\prime}, \Gamma \Rightarrow \Delta, m / h: \mathcal{P} A, m / h^{\prime}: A}{m \in h^{\prime}, \Gamma \Rightarrow \Delta, m / h: \mathcal{P} A} R \mathcal{P}$ $\frac{i=i, \Gamma \Rightarrow \Delta}{\Gamma \Rightarrow \Delta}$ Refl $_{=} \quad \frac{j=k, i=j, i=k, \Gamma \Rightarrow \Delta}{i=j, i=k, \Gamma \Rightarrow \Delta}$ Etrans $_{=}$ $\frac{i=j, A t(i), A t(j), \Gamma \Rightarrow \Delta}{i=j, A t(i), \Gamma \Rightarrow \Delta} \operatorname{Repl}_{A t} \quad \frac{h \sim_{m}^{i} h, \Gamma \Rightarrow \Delta}{\Gamma \Rightarrow \Delta} \operatorname{Refl}_{\sim_{m}^{i}}$ $\frac{h_{2} \sim_{m}^{i} h_{3}, h_{1} \sim_{m}^{i} h_{2}, h_{1} \sim_{m}^{i} h_{3}, \Gamma \Rightarrow \Delta}{h_{1} \sim_{m}^{i} h_{2}, h_{1} \sim_{m}^{i} h_{3}, \Gamma \Rightarrow \Delta}{\text { Etrans } \sim_{m}^{i}}$ $\frac{m \in h, h \sim_{m}^{i} h^{\prime}, \Gamma \Rightarrow \Delta}{h \sim_{m}^{i} h^{\prime}, \Gamma \Rightarrow \Delta} W D$

$\frac{\left\{i_{l} \neq i_{m}\right\}_{1 \leq l<m \leq k}, \Gamma \Rightarrow \Delta}{\operatorname{Diff}\left(i_{1}, \ldots, i_{k}\right), \Gamma \Rightarrow \Delta} \operatorname{Diff}_{k}$ $\overline{i \neq j, i=j, \Gamma \Rightarrow \Delta} \neq$ $\frac{h \sim_{m}^{i_{1}} h_{1}, \ldots, h \sim_{m}^{i_{n}} h_{n}, \operatorname{Diff}\left(i_{1}, \ldots, i_{k}\right), m \in h_{1}, \ldots, m \in h_{n}, h_{1} \sim_{m}^{i_{1}} h_{1}^{\prime}, \ldots, h_{n} \sim_{m}^{i_{n}} h_{n}^{\prime}, \Gamma \Rightarrow \Delta}{\operatorname{Diff}\left(i_{1}, \ldots, i_{k}\right), m \in h_{1}, \ldots, m \in h_{n}, h_{1} \sim_{m}^{i_{1}} h_{1}^{\prime}, \ldots, h_{n} \sim_{m}^{i_{n}} h_{n}^{\prime}, \Gamma \Rightarrow \Delta}$ Ind $_{k}$

Application conditions: $h^{\prime}$ is fresh in $\mathrm{R} \square^{i}, \mathrm{RS}, \mathrm{LP}$ and $I n d_{k}$, and $1 \leq n \leq k$ in $\operatorname{Ind}_{k}$

Fig. 1 G3DSTIT 
$\mathbf{A 2} \mathcal{D}^{i} A \supset \mathcal{D}^{i} \mathcal{D}^{i} A$.

\section{Proof}

(1)

$$
\begin{aligned}
& \underline{h^{\prime \prime} \sim_{m}^{i} h, h^{\prime \prime} \sim_{m}^{i} h^{\prime}, h^{\prime} \sim_{m}^{i} h, m / h: \square^{i} A, m / h^{\prime \prime}: A \Rightarrow m / h^{\prime \prime}: A, m / h: \mathcal{S} A} \\
& \frac{h^{\prime \prime} \sim_{m}^{i} h, h^{\prime \prime} \sim_{m}^{i} h^{\prime}, h^{\prime} \sim_{m}^{i} h, m / h: \square^{i} A \Rightarrow m / h^{\prime \prime}: A, m / h: \mathcal{S} A}{h^{\prime \prime} \sim_{m}^{i} h^{\prime}, h^{\prime} \sim_{m}^{i} h, m / h: \square^{i} A \Rightarrow m / h^{\prime \prime}: A, m / h: \mathcal{S} A} \text { Erans }_{\sim_{m}^{i}}^{i} \\
& h^{\prime} \sim_{m}^{i} h, m / h: \square^{i} A \Rightarrow m / h^{\prime}: \square^{i} A, m / h: \mathcal{S} A \quad R \square^{i}
\end{aligned}
$$

(2)

$$
\begin{gathered}
\frac{m / h^{\prime \prime \prime}: A, m \in h^{\prime \prime \prime}, m / h^{\prime}: \mathcal{S} A, h^{\prime} \sim_{m}^{i} h, m / h: \square^{i} A \Rightarrow m / h^{\prime \prime \prime}: A}{\frac{m \in h^{\prime \prime \prime}, m / h^{\prime}: \mathcal{S} A, h^{\prime} \sim_{m}^{i} h, m / h: \square^{i} A \Rightarrow m / h^{\prime \prime \prime}: A}{m / h^{\prime}: \mathcal{S} A, h^{\prime} \sim_{m}^{i} h, m / h: \square^{i} A \Rightarrow m / h: \mathcal{S} A}} R \mathcal{S} \\
\frac{h^{\prime} \sim_{m}^{i} h, m / h: \square^{i} A \Rightarrow m / h^{\prime}: \mathcal{D}^{i} A, m / h: \mathcal{S} A}{m / h: \square^{i} A \Rightarrow m / h: \square^{i} \mathcal{D}^{i} A, m / h: \mathcal{S} A} R \square^{i}
\end{gathered}
$$

(3)

$$
\begin{aligned}
& m / h^{\prime}: A, h^{\prime} \sim_{m}^{i} h^{\prime}, m / h^{\prime}: \square^{i} A, m \in h^{\prime}, m / h: \mathcal{S D}^{i} A, m / h: \square^{i} A \Rightarrow m / h^{\prime}: A, m / h^{\prime}: \mathcal{S} A \\
& h^{\prime} \sim_{m}^{i} h^{\prime}, m / h^{\prime}: \square^{i} A, m \in h^{\prime}, m / h: \mathcal{S D}^{i} A, m / h: \square^{i} A \Rightarrow m / h^{\prime}: A, m / h^{\prime}: \mathcal{S} A \operatorname{Refl}_{\sim_{m}^{i}} \\
& \underline{m / h^{\prime}: \square^{i} A, m \in h^{\prime}, m / h: \mathcal{S} \mathcal{D}^{i} A, m / h: \square^{i} A \Rightarrow m / h^{\prime}: A, m / h^{\prime}: \mathcal{S} A} L_{\mathcal{D}^{i}} \\
& \underline{m / h^{\prime}: \mathcal{D}^{i} A, m \in h^{\prime}, m / h: \mathcal{S D}^{i} A, m / h: \square^{i} A \Rightarrow m / h^{\prime}: A} L \mathcal{S} \\
& \frac{m \in h^{\prime}, m / h: \mathcal{S D}^{i} A, m / h: \square^{i} A \Rightarrow m / h^{\prime}: A}{m / h: \mathcal{S D}^{i} A, m / h: \square^{i} A \Rightarrow m / h: \mathcal{S} A} R \mathcal{S}
\end{aligned}
$$

$$
\begin{gathered}
\frac{m / h: \square^{i} A \Rightarrow m / h: \mathcal{D}^{i} \mathcal{D}^{i} A, m / h: \mathcal{S A}}{m / h: \mathcal{D}^{i} A \Rightarrow m / h: \mathcal{D}^{i} \mathcal{D}^{i} A} \\
R \mathcal{D}^{i}
\end{gathered}
$$

$\mathbf{A 3} \mathcal{D}^{i} A \supset \mathcal{D}^{i} \neg \mathcal{D}^{i} \neg \mathcal{D}^{i} A$.

Proof Proposition* 5.4.

A4 $\left(\mathcal{D}^{i} A \& \mathcal{D}^{i}(B \& C)\right) \supset \mathcal{D}^{i}(A \& C)$.

\section{Proof}

(1)

$$
\begin{gathered}
\frac{h^{\prime} \sim_{m}^{i} h, m / h^{\prime}: A, \ldots \Rightarrow m / h^{\prime}: A, \ldots}{\frac{h^{\prime} \sim_{m}^{i} h, m / h: \square^{i} A, \ldots \Rightarrow m / h^{\prime}: A, \ldots}{h^{\prime} \sim_{m}^{i} h, m / h: \mathcal{D}^{i} A, \ldots \Rightarrow m / h^{\prime}: A} L \square^{i}} L \mathcal{D}^{i} \quad \frac{h^{\prime} \sim_{m}^{i} h, \ldots, m / h^{\prime}: B, m / h^{\prime}: C \Rightarrow m / h^{\prime}: C, \ldots}{h^{\prime} \sim_{m}^{i} h, \ldots, m / h^{\prime}: B \& C \Rightarrow m / h^{\prime}: C, \ldots} L \& \\
\frac{h^{\prime} \sim_{m}^{i} h, \ldots, m / h: \square^{i}(B \& C) \Rightarrow m / h^{\prime}: C, \ldots}{h^{\prime} \sim_{m}^{i} h, \ldots, m / h: \mathcal{D}^{i}(B \& C) \Rightarrow m / h^{\prime}: C} L \square^{i} \\
\frac{h^{\prime} \sim_{m}^{i} h, m / h: \mathcal{D}^{i} A, m / h: \mathcal{D}^{i}(B \& C) \Rightarrow m / h^{\prime}: A \& C}{m / h: \mathcal{D}^{i} A, m / h: \mathcal{D}^{i}(B \& C) \Rightarrow m / h: \square^{i}(A \& C)} R^{\prime} \square^{i}
\end{gathered}
$$


(2)

$$
\begin{aligned}
& \underline{m / h^{\prime}: A, m / h^{\prime}: C, m \in h^{\prime}, m / h: \mathcal{S}(A \& C), m / h: \square^{i} A, m / h: \mathcal{D}^{i}(B \& C) \Rightarrow m / h^{\prime}: A} \underset{ }{L} \& \\
& m / h^{\prime}: A \& C, m \in h^{\prime}, m / h: \mathcal{S}(A \& C), m / h: \square^{i} A, m / h: \mathcal{D}^{i}(B \& C) \Rightarrow m / h^{\prime}: A
\end{aligned}
$$

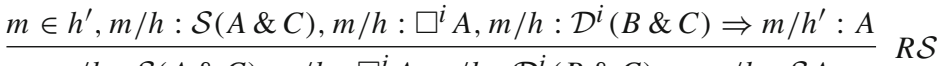

$$
\begin{aligned}
& \frac{m / h: \mathcal{S}(A \& C), m / h: \square^{i} A, m / h: \mathcal{D}^{i}(B \& C) \Rightarrow m / h: \mathcal{S} A}{m / h: \mathcal{S}(A \& C), m / h: \mathcal{D}^{i} A, m / h: \mathcal{D}^{i}(B \& C) \Rightarrow} L \mathcal{D}^{i}
\end{aligned}
$$

$$
\frac{\overline{m / h: \mathcal{D}^{i} A, m / h: \mathcal{D}^{i}(B \& C) \Rightarrow m / h: \mathcal{D}^{i}(A \& C)}}{m / h: \mathcal{D}^{i} A \& \mathcal{D}^{i}(B \& C) \Rightarrow m / h: \mathcal{D}^{i}(A \& C)} L \&
$$

$\mathbf{A 5}\left(\mathcal{D}^{i} A \& \neg \mathcal{D}^{i} B\right) \supset \mathcal{D}^{i}\left(A \& \neg \mathcal{D}^{i} B\right)$.

\section{Proof}

(1)

$$
\begin{aligned}
& h^{\prime} \sim_{m}^{i} h, m / h: \square^{i} A, m / h^{\prime}: A \Rightarrow m / h^{\prime}: A, \ldots \quad \begin{array}{l}
\text { Lemma* } \\
h^{\prime}
\end{array} \\
& \frac{\frac{h^{\prime} \sim_{m}^{i} h, m / h: \square^{i} A \Rightarrow m / h^{\prime}: A, \ldots}{h^{\prime} \sim_{m}^{i} h, m / h: \mathcal{D}^{i} A \Rightarrow m / h^{\prime}: A, \ldots} L \mathcal{D}^{i} \quad L \square^{i} \quad \frac{m / h^{\prime}: \mathcal{D}^{i} B, h^{\prime} \sim_{m}^{i} h, m / h: \mathcal{D}^{i} A \Rightarrow m / h: \mathcal{D}^{i} B}{h^{\prime} \sim_{m}^{i} h, m / h: \mathcal{D}^{i} A \Rightarrow m / h^{\prime}: \neg \mathcal{D}^{i} B, m / h: \mathcal{D}^{i} B}}{R \neg} R
\end{aligned}
$$

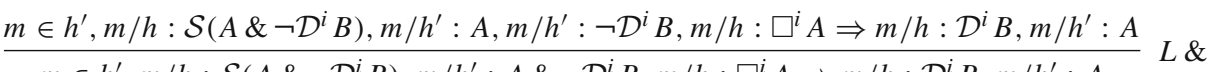

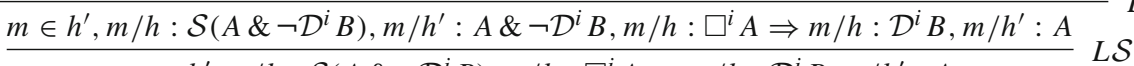

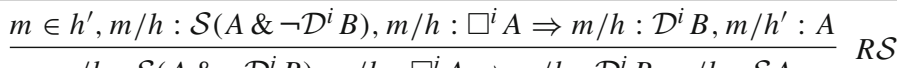

$$
\begin{aligned}
& \frac{m / h: \mathcal{S}\left(A \& \neg \mathcal{D}^{i} B\right), m / h: \square^{i} A \Rightarrow m / h: \mathcal{D}^{i} B, m / h: \mathcal{S} A}{m / h: \mathcal{S}\left(A \& \neg \mathcal{D}^{i} B\right), m / h: \mathcal{D}^{i} A \Rightarrow m / h: \mathcal{D}^{i} B} L \mathcal{D}^{i}
\end{aligned}
$$

$\frac{\frac{m / h: \mathcal{D}^{i} A \Rightarrow m / h: \mathcal{D}^{i}\left(A \& \neg \mathcal{D}^{i} B\right), m / h: \mathcal{D}^{i} B}{m / h: \mathcal{D}^{i} A, m / h: \neg \mathcal{D}^{i} B \Rightarrow m / h: \mathcal{D}^{i}\left(A \& \neg \mathcal{D}^{i} B\right)}}{\frac{m}{m / h: \mathcal{D}^{i} A \& \neg \mathcal{D}^{i} B \Rightarrow m / h: \mathcal{D}^{i}\left(A \& \neg \mathcal{D}^{i} B\right)}} L \&$

A6 $\mathcal{D}^{i}\left(\neg \mathcal{D}^{i} A \&\left(B \& \neg \mathcal{D}^{i} B\right)\right) \supset \subset \mathcal{D}^{i} \neg \mathcal{D}^{i}\left(A \&\left(B \& \neg \mathcal{D}^{i} B\right)\right)$

\section{Proof See "Appendix A".}

This concludes the proof of adequacy of G3DSTIT for the logic $\mathrm{L}_{0}$. Close inspection of the rules shows that the rule of independence of agents was not required. This will not be the case for the next system, where the full G3DSTIT will be used. 


\section{Logic $L_{1}$}

Logic $\mathrm{L}_{1}$ can be divided into three separate segments - those axioms concerning identity, the one that deals with independence of agents, and the remaining axioms. The first group needs to be addressed separately due to the specificities of identity in G3, while the second warrants a separate section due to sheer complexity.

\subsection{Identity Axioms}

The first two axioms deal with the standard properties of identity, each axiom corresponds to a rule and both are shown straightforwardly. We will discuss the third of these axioms in greater detail, however.

A7 $i=i$

A8 $i=j \supset(A \supset A(i / j))$.

Proof Both of these axioms are proven by Proposition* 3.1.

A9 $\mathcal{D}^{i} A \supset \subset\left(\mathcal{D}^{i}(A \& j=k) \vee \mathcal{D}^{i}(A \& j \neq k)\right)$.

Proof We first note that $i=j$ and $i \neq j$ are relational atoms and don't occur labelled by points, since identity is taken as rigid. Since the proof of this axiom requires labelled identity atoms, we can express rigidity in a form of a rule as in Negri and Orlandelli (2019):

$$
\frac{m \in h^{\prime}, m / h^{\prime}: j=k, m / h: j=k, \Gamma \Rightarrow \Delta}{m \in h^{\prime}, m / h: j=k, \Gamma \Rightarrow \Delta} \operatorname{Rig}
$$

and thereby we can derive

$$
\frac{m / h^{\prime \prime}: j=k, m \in h^{\prime \prime}, m / h^{\prime}: j=k, \Gamma \Rightarrow \Delta, m / h^{\prime}: j=k}{\frac{m \in h^{\prime \prime}, m / h^{\prime}: j=k, \Gamma \Rightarrow \Delta, m / h^{\prime}: j=k}{m / h^{\prime}: j=k, \Gamma \Rightarrow \Delta, m / h^{\prime \prime}: j=k}} \text { Rig }
$$

We now proceed with the proof. Left to right:

(1)

$$
\frac{m / h^{\prime}: j=k, h^{\prime} \sim_{m}^{i} h \sim_{m}^{i} h^{\prime \prime}, m / h: \square^{i} A \Rightarrow m / h^{\prime \prime}: j=k, m / h: \mathcal{S} A}{h^{\prime} \sim_{m}^{i} h \sim_{m}^{i} h^{\prime \prime}, m / h: \square^{i} A \Rightarrow m / h^{\prime \prime}: j=k, m / h^{\prime}: j \neq k, m / h: \mathcal{S} A} R \neg
$$

$$
\begin{gathered}
\frac{h{ }_{m}^{i} h^{\prime \prime}, m / h: \square^{i} A \Rightarrow m / h^{\prime \prime}: A, \ldots}{(1)} \\
\frac{h^{\prime} \sim_{m}^{i} h, m / h: \square^{i} A \Rightarrow \ldots, m / h^{\prime}: A, \ldots}{\frac{h^{\prime} \sim_{m}^{i} h \sim_{m}^{i} h^{\prime \prime}, m / h: \square^{i} A \Rightarrow m / h^{\prime \prime}: A \& j=k, m / h^{\prime}: j \neq k, m / h: \mathcal{S} A}{h^{\prime} \sim_{m}^{i} h, m / h: \square^{i} A \Rightarrow m / h: \square^{i}(A \& j=k), m / h^{\prime}: j \neq k, m / h: \mathcal{S} A}} R \square^{i} \\
\frac{h^{\prime} \sim_{m}^{i} h, m / h: \square^{i} A \Rightarrow m / h: \square^{i}(A \& j=k), m / h^{\prime}: A \& j \neq k, m / h: \mathcal{S} A}{m / h: \square^{i} A \Rightarrow m / h: \square^{i}(A \& j=k), m / h: \square^{i}(A \& j \neq k), m / h: \mathcal{S} A} R \square^{i}
\end{gathered}
$$

$\frac{\frac{m / h: \mathcal{S}(A \& j \neq k), \ldots \Rightarrow \ldots, m / h: \mathcal{S} A}{m / h: \square^{i} A \Rightarrow m / h: \mathcal{D}^{i}(A \& j=k), m / h: \square^{i}(A \& j \neq k), m / h: \mathcal{S} A} R \mathcal{D}^{i} m / h: \mathcal{S}(A \& j=k), \ldots \Rightarrow \ldots, m / h: \mathcal{S} A}{\frac{m / h: \square^{i} A \Rightarrow m / h: \mathcal{D}^{i}(A \& j=k), m / h: \mathcal{D}^{i}(A \& j \neq k), m / h: \mathcal{S} A}{\frac{m / h: \mathcal{D}^{i} A \Rightarrow m / h: \mathcal{D}^{i}(A \& j=k), m / h: \mathcal{D}^{i}(A \& j \neq k)}{m / h: \mathcal{D}^{i} A \Rightarrow m / h: \mathcal{D}^{i}(A \& j=k) \vee \mathcal{D}^{i}(A \& j \neq k)} R} R}$ 
Right to left:

(1a)

$$
\begin{aligned}
& m / h^{\prime}: A, m / h^{\prime}: j=k, h^{\prime} \sim_{m}^{i} h, m / h: \square^{i}(A \& j=k) \Rightarrow m / h^{\prime}: A, m / h: \mathcal{S}(A \& j=k) \\
& \frac{m / h^{\prime}: A \& j=k, h^{\prime} \sim_{m}^{i} h, m / h: \square^{i}(A \& j=k) \Rightarrow m / h^{\prime}: A, m / h: \mathcal{S}(A \& j=k)}{h^{\prime} \sim_{m}^{i} h, m / h: \square^{i}(A \& j=k) \Rightarrow m / h^{\prime}: A, m / h: \mathcal{S}(A \& j=k)} L \square^{i} \\
& \frac{h^{\prime} \sim_{m}^{i} h, m / h: \square^{i}(A \& j=k) \Rightarrow m / h^{\prime}: A, m / h: \mathcal{S}(A \& j=k)}{m / h: \square^{i}(A \& j=k) \Rightarrow m / h: \square^{i} A, m / h: \mathcal{S}(A \& j=k)} R \square^{i}
\end{aligned}
$$

(1b)

$$
\frac{m / h: A, m / h: j=k, h \sim_{m}^{i} h, m \in h^{\prime}, m / h: \mathcal{S} A, m / h: \square^{i}(A \& j=k) \Rightarrow m / h^{\prime}: j=k}{\frac{m / h: A \& j=k, h \sim_{m}^{i} h, m \in h^{\prime}, m / h: \mathcal{S} A, m / h: \square^{i}(A \& j=k) \Rightarrow m / h^{\prime}: j=k}{2}} L \&
$$

(1c)

$$
\frac{\frac{m / h^{\prime}: A, m \in h^{\prime}, m / h: \mathcal{S} A, \ldots \Rightarrow m / h^{\prime}: A}{m \in h^{\prime}, m / h: \mathcal{S} A, m / h: \square^{i}(A \& j=k) \Rightarrow m / h^{\prime}: A} L \mathcal{S} \quad(1 b)}{\frac{m \in h^{\prime}, m / h: \mathcal{S} A, m / h: \square^{i}(A \& j=k) \Rightarrow m / h^{\prime}: A \& j=k}{m / h: \mathcal{S} A, m / h: \square^{i}(A \& j=k) \Rightarrow m / h: \mathcal{S}(A \& j=k)} R \mathcal{S}}
$$

(1)

$$
\frac{(1 a)}{\frac{m / h: \square^{i}(A \& j=k) \Rightarrow m / h: \mathcal{D}^{i} A, m / h: \mathcal{S}(A \& j=k)}{m / h: \mathcal{D}^{i}(A \& j=k) \Rightarrow m / h: \mathcal{D}^{i} A}} R \mathcal{D}^{i}
$$

(2) very similar to (1)

(1)

$$
\overline{m / h: \mathcal{D}^{i}(A \& j=k) \vee \mathcal{D}^{i}(A \& j \neq k) \Rightarrow m / h: \mathcal{D}^{i} A} R \vee
$$

Of course, one should only see this proof as a sketch of why the axiom is intuitively acceptable. Since neither $j=k$ nor $j \neq k$ occur in the succedent (on the pain of loss of admisibility of cut, as discussed in Negri and Pavlović 2020), this is not a proof in G3DSTIT, even with the rule for rigidity added. What this axiom states, namely that $j=k$ is either settled true or settled false Xu (1998, p. 515), is instead expressed by using $j=k$ and $j \neq k$ as a relational atom.

\subsection{Remaining Axioms}

$\mathbf{A 1 0}\left(\mathcal{D}^{i} A \& \mathcal{D}^{k}\left(B \&\left(C \& \neg \mathcal{D}^{k} C\right)\right)\right) \supset \mathcal{D}^{i}\left(A \&\left(C \& \neg \mathcal{D}^{i} C\right)\right)$.

We begin this proof with a lemma:

Lemma 4.1 $m / h: \mathcal{D}^{k}\left(B \&\left(C \& \neg \mathcal{D}^{k} C\right)\right) \Rightarrow m / h^{\prime}: C$. 
Proof We omit applications of WD for brevity.

$$
\begin{aligned}
& \frac{\ldots, m / h^{\prime \prime}: C, \ldots \Rightarrow \ldots, m / h^{\prime \prime}: C}{h^{\prime \prime} \sim_{m}^{k} h, \ldots, m / h^{\prime \prime}: B \&\left(C \& \neg \mathcal{D}^{k} C\right) \Rightarrow \ldots, m / h^{\prime \prime}: C} L \& \\
& \frac{\frac{h^{\prime \prime} \sim_{m}^{k} h, \ldots, m / h^{\prime \prime}: B \&\left(C \& \neg \mathcal{D}^{k} C\right) \Rightarrow \ldots, m / h^{\prime \prime}: C}{h^{\prime \prime} h, \ldots, m / h: \square^{k}\left(B \&\left(C \& \neg \mathcal{D}^{k} C\right)\right) \Rightarrow \ldots, m / h^{\prime \prime}: C}}{\ldots, m / h: \square^{k}\left(B \&\left(C \& \neg \mathcal{D}^{k} C\right)\right) \Rightarrow \ldots, m / h: \square^{k} C} R \square^{k} \frac{m / h^{\prime}: C, \ldots \Rightarrow m / h^{\prime}: C, \ldots}{m / h: \mathcal{S} C, \ldots \Rightarrow m / h^{\prime}: C, \ldots} L \mathcal{S} \\
& \frac{\ldots, m / h: \square^{k}\left(B \&\left(C \& \neg \mathcal{D}^{k} C\right)\right) \Rightarrow \ldots, m / h: \square^{k} C}{m / h: B, m / h: C, h \sim_{m}^{k} h, m / h: \square^{k}\left(B \&\left(C \& \neg \mathcal{D}^{k} C\right)\right) \Rightarrow m / h^{\prime}: C, \ldots, m / h: \mathcal{D}^{k} C} R \mathcal{D}^{k} \\
& m / h: B, m / h: C, m / h: \neg \mathcal{D}^{k} C, h \sim_{m}^{k} h, m / h: \square^{k}\left(B \&\left(C \& \neg \mathcal{D}^{k} C\right)\right) \Rightarrow m / h^{\prime}: C, \ldots \\
& m / h: B \&\left(C \& \neg \mathcal{D}^{k} C\right), h \sim_{m}^{k} h, m / h: \square^{k}\left(B \&\left(C \& \neg \mathcal{D}^{k} C\right)\right) \Rightarrow m / h^{\prime}: C, \ldots \quad L \& \\
& \overline{h \sim_{m}^{k} h, m / h: \square^{k}\left(B \&\left(C \& \neg \mathcal{D}^{k} C\right)\right) \Rightarrow m / h^{\prime}: C, \ldots} L \square^{k} \\
& \overline{m / h: \square^{k}\left(B \&\left(C \& \neg \mathcal{D}^{k} C\right)\right) \Rightarrow m / h^{\prime}: C, m / h: \mathcal{S}\left(B \&\left(C \& \neg \mathcal{D}^{k} C\right)\right)} \operatorname{Refl}_{\sim_{m}^{k}}^{k} \\
& m / h: \mathcal{D}^{k}\left(B \&\left(C \& \neg \mathcal{D}^{k} C\right)\right) \Rightarrow m / h^{\prime}: C
\end{aligned}
$$

\section{Proof}

(1a)

\section{Lemma 4.1}

$m \in h^{\prime \prime}, m / h^{\prime}: \square^{i} C, h^{\prime} \sim_{m}^{i} h, m / h: \mathcal{D}^{i} A, m / h: \mathcal{D}^{k}\left(B \&\left(C \& \neg \mathcal{D}^{k} C\right)\right) \Rightarrow m / h^{\prime \prime}: C$ $m / h^{\prime}: \square^{i} C, h^{\prime} \sim_{m}^{i} h, m / h: \mathcal{D}^{i} A, m / h: \mathcal{D}^{k}\left(B \&\left(C \& \neg \mathcal{D}^{k} C\right)\right) \Rightarrow m / h^{\prime}: \mathcal{S} C$

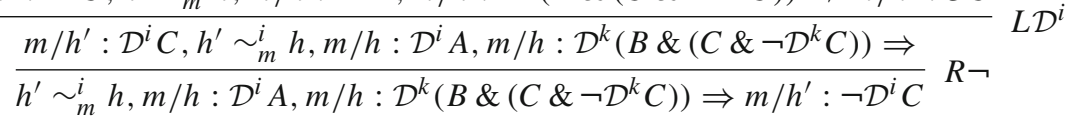

(1b)

\section{Lemma 4.1}

$\overline{h^{\prime} \sim{ }_{m}^{i} h, m / h: \mathcal{D}^{i} A, m / h: \mathcal{D}^{k}\left(B \&\left(C \& \neg \mathcal{D}^{k} C\right)\right) \Rightarrow m / h^{\prime}: C \& \neg \mathcal{D}^{i} C} R \&$

(1c)

$$
\begin{aligned}
& h^{\prime} \sim_{m}^{i} h, m / h: \square^{i} A, m / h^{\prime}: A, m / h: \mathcal{D}^{k}\left(B \&\left(C \& \neg \mathcal{D}^{k} C\right)\right) \Rightarrow m / h^{\prime}: A, m / h: \mathcal{S} A \\
& \frac{h^{\prime} \sim_{m}^{i} h, m / h: \square^{i} A, m / h: \mathcal{D}^{k}\left(B \&\left(C \& \neg \mathcal{D}^{k} C\right)\right) \Rightarrow m / h^{\prime}: A, m / h: \mathcal{S} A}{h^{\prime} \sim_{m}^{i} h, m / h: \mathcal{D}^{i} A, m / h: \mathcal{D}^{k}\left(B \&\left(C \& \neg \mathcal{D}^{k} C\right)\right) \Rightarrow m / h^{\prime}: A} L \mathcal{D}^{\prime} \\
& m / h: \mathcal{D}^{i} A, m / h: \mathcal{D}^{k}\left(B \&\left(C \& \neg \mathcal{D}^{k} C\right)\right) \Rightarrow m / h: \square^{i}\left(A \&\left(C \& \neg \mathcal{D}^{i} C\right)\right) \quad R \square \\
& m \in h^{\prime}, m / h^{\prime}: A, \ldots \Rightarrow m / h^{\prime}: A \\
& \frac{m \in h^{\prime}, m / h^{\prime}: A \&\left(C \& \neg \mathcal{D}^{i} C\right), \ldots, m / h: \square^{i} A, \ldots \Rightarrow m / h^{\prime}: A}{m \in h^{\prime}, m / h: \mathcal{S}\left(A \&\left(C \& \neg \mathcal{D}^{i} C\right)\right), m / h: \square^{i} A, \ldots \Rightarrow m / h^{\prime}: A} L \mathcal{S} \\
& m / h: \mathcal{S}\left(A \&\left(C \& \neg \mathcal{D}^{i} C\right)\right), m / h: \square^{i} A, \ldots \Rightarrow m / h: \mathcal{S} A \quad R \mathcal{S} \\
& \text { (1c) } \overline{m / h: \mathcal{S}\left(A \&\left(C \& \neg \mathcal{D}^{i} C\right)\right), m / h: \mathcal{D}^{i} A, m / h: \mathcal{D}^{k}\left(B \&\left(C \& \neg \mathcal{D}^{k} C\right)\right) \Rightarrow} L \mathcal{D}^{i} \\
& m / h: \mathcal{D}^{i} A, m / h: \mathcal{D}^{k}\left(B \&\left(C \& \neg \mathcal{D}^{k} C\right)\right) \Rightarrow m / h: \mathcal{D}^{i}\left(A \&\left(C \& \neg \mathcal{D}^{i} C\right)\right) \\
& m / h: \mathcal{D}^{i} A \& \mathcal{D}^{k}\left(B \&\left(C \& \neg \mathcal{D}^{k} C\right)\right) \Rightarrow m / h: \mathcal{D}^{i}\left(A \&\left(C \& \neg \mathcal{D}^{i} C\right)\right)
\end{aligned}
$$


$\mathbf{A 1 1}\left(\mathcal{D}^{i} A \& \neg \mathcal{D}^{i}\left(A \& \neg \mathcal{D}^{k} B\right)\right) \supset\left(\mathcal{D}^{k} B \vee \mathcal{D}^{k} \neg \mathcal{D}^{k} B\right)$.

\section{Proof}

(1a)

\section{Lemma* 5.1}

$$
\frac{\frac{m / h^{\prime \prime}: \mathcal{D}^{k} B, h^{\prime \prime} \sim_{m}^{k} h, m / h^{\prime}: \mathcal{D}^{k} B, h^{\prime} \sim_{m}^{i} h, m / h: \square^{i} A \Rightarrow m / h: \mathcal{D}^{k} B, m / h: \mathcal{S} A}{h^{\prime \prime} \sim_{m}^{k} h, m / h^{\prime}: \mathcal{D}^{k} B, h^{\prime} \sim_{m}^{i} h, m / h: \square^{i} A \Rightarrow m / h: \mathcal{D}^{k} B, m / h^{\prime \prime}: \neg \mathcal{D}^{k} B, m / h: \mathcal{S} A}}{m^{\prime} h^{\prime}: \mathcal{D}^{k} B, h^{\prime} \sim_{m}^{i} h, m / h: \square^{i} A \Rightarrow m / h: \mathcal{D}^{k} B, m / h: \square^{k} \neg \mathcal{D}^{k} B, m / h: \mathcal{S} A} R \square^{k}
$$

\section{Lemma* 5.1}

$$
\begin{aligned}
& \text { (1a) } \frac{\frac{\ldots, m / h^{\prime}: \mathcal{D}^{k} B, h^{\prime} \sim_{m}^{i} h, m / h: \square^{i} A \Rightarrow m / h: \mathcal{D}^{k} B, m / h: \mathcal{S} A, m / h^{\prime}: \mathcal{D}^{k} B}{m / h^{\prime}: \neg \mathcal{D}^{k} B, \ldots, m / h^{\prime}: \mathcal{D}^{k} B, h^{\prime} \sim_{m}^{i} h, m / h: \square^{i} A \Rightarrow m / h: \mathcal{D}^{k} B, m / h: \mathcal{S} A}}{m / h: \mathcal{S} \neg \mathcal{D}^{k} B, m / h^{\prime}: \mathcal{D}^{k} B, h^{\prime} \sim_{m}^{i} h, m / h: \square^{i} A \Rightarrow m / h: \mathcal{D}^{k} B, m / h: \mathcal{S} A} \text { WD, LS } \\
& \frac{m / h^{\prime}: \mathcal{D}^{k} B, h^{\prime} \sim_{m}^{i} h, m / h: \square^{i} A \Rightarrow m / h: \mathcal{D}^{k} B, m / h: \mathcal{D}^{k} \neg \mathcal{D}^{k} B, m / h: \mathcal{S} A}{h^{\prime} \sim{ }_{m}^{i} h, m / h: \square^{i} A \Rightarrow m / h: \mathcal{D}^{k} B, m / h: \mathcal{D}^{k} \neg \mathcal{D}^{k} B, m / h: \mathcal{S} A, m / h^{\prime}: \neg \mathcal{D}^{k} B} R \neg
\end{aligned}
$$

$$
\begin{aligned}
& h^{\prime} \sim_{m}^{i} h, \ldots, m / h^{\prime}: A \Rightarrow m / h: \mathcal{D}^{k} B, m / h: \mathcal{D}^{k} \neg \mathcal{D}^{k} B, m / h: \mathcal{S} A, m / h^{\prime}: A \\
& \frac{\frac{h^{\prime} \sim_{m}^{i} h, m / h: \square^{i} A \Rightarrow m / h: \mathcal{D}^{k} B, m / h: \mathcal{D}^{k} \neg \mathcal{D}^{k} B, m / h: \mathcal{S} A, m / h^{\prime}: A}{h^{\prime} \sim{ }_{m}^{i} h, m / h: \square^{i} A \Rightarrow m / h: \mathcal{D}^{k} B, m / h: \mathcal{D}^{k} \neg \mathcal{D}^{k} B, m / h: \mathcal{S} A, m / h^{\prime}: A \& \neg \mathcal{D}^{k} B}}{.} R \& \\
& m / h: \square^{i} A \Rightarrow m / h: \mathcal{D}^{k} B, m / h: \mathcal{D}^{k} \neg \mathcal{D}^{k} B, m / h: \mathcal{S} A, m / h: \square^{i}\left(A \& \neg \mathcal{D}^{k} B\right)
\end{aligned}
$$

$$
\begin{aligned}
& \underline{m \in h^{\prime}, \ldots, m / h^{\prime}: A, m / h^{\prime}: \neg \mathcal{D}^{k} B, m / h: \square^{i} A \Rightarrow m / h: \mathcal{D}^{k} B, m / h: \mathcal{D}^{k} \neg \mathcal{D}^{k} B, m / h^{\prime}: A} \\
& \frac{m \in h^{\prime}, \ldots, m / h^{\prime}: A \& \neg \mathcal{D}^{k} B, m / h: \square^{i} A \Rightarrow m / h: \mathcal{D}^{k} B, m / h: \mathcal{D}^{k} \neg \mathcal{D}^{k} B, m / h^{\prime}: A}{m \in h^{\prime}} L \mathcal{S} \\
& \text { (1c) } \\
& \underline{m \in h^{\prime}, m / h: \mathcal{S}\left(A \& \neg \mathcal{D}^{k} B\right), m / h: \square^{i} A \Rightarrow m / h: \mathcal{D}^{k} B, m / h: \mathcal{D}^{k} \neg \mathcal{D}^{k} B, m / h^{\prime}: A} \begin{array}{l}
L \mathcal{S} \\
R \mathcal{S}
\end{array} \\
& \frac{m / h: \mathcal{S}\left(A \& \neg \mathcal{D}^{k} B\right), m / h: \square^{i} A \Rightarrow m / h: \mathcal{D}^{k} B, m / h: \mathcal{D}^{k} \neg \mathcal{D}^{k} B, m / h: \mathcal{S} A}{m / h: \square^{i} A \Rightarrow m / h: \mathcal{D}^{k} B, m / h: \mathcal{D}^{k} \neg \mathcal{D}^{k} B, m / h: \mathcal{S} A, m / h: \mathcal{D}^{i}\left(A \& \neg \mathcal{D}^{k} B\right)} R \mathcal{D}^{i} \\
& \underline{m / h: \square^{i} A, m / h: \neg \mathcal{D}^{i}\left(A \& \neg \mathcal{D}^{k} B\right) \Rightarrow m / h: \mathcal{D}^{k} B, m / h: \mathcal{D}^{k} \neg \mathcal{D}^{k} B, m / h: \mathcal{S} A} \quad L \neg \mathcal{D}^{i} \\
& \frac{m / h: \mathcal{D}^{i} A, m / h: \neg \mathcal{D}^{i}\left(A \& \neg \mathcal{D}^{k} B\right) \Rightarrow m / h: \mathcal{D}^{k} B, m / h: \mathcal{D}^{k} \neg \mathcal{D}^{k} B}{m / h: \mathcal{D}^{i} A \& \neg \mathcal{D}^{i}\left(A \& \neg \mathcal{D}^{k} B\right) \Rightarrow m / h: \mathcal{D}^{k} B \vee \mathcal{D}^{k} \neg \mathcal{D}^{k} B} \quad R \vee L \&
\end{aligned}
$$

$\mathbf{A 1 2} \mathcal{D}^{i} \neg \mathcal{D}^{k} B \supset i=k$.

Proof By contraposition from the second claim in Theorem* 5.2.

\subsection{Axioms AIA}

While the axiom schema $\mathrm{AIA}_{n}$ is defined for any $2 \leq n$, only the simplest case of $n=2$ will suffice for the demonstration, as the proofs for any other $n$ differ in size but not in substance. We begin the proof with a pair of lemmas: 
Lemma $4.2 a=b_{1}, \operatorname{Diff}\left(b_{1}, b_{2}\right), h^{2} \sim_{m}^{a} h, h^{1} \sim_{m}^{a} h, m / h: \mathcal{D}^{a}\left(A \& \neg\left(\mathcal{D}^{b_{1}} B_{1} \& \mathcal{D}^{b_{2}} B_{2}\right)\right)$, $m / h^{1}: \mathcal{D}^{b_{1}} B_{1}, m / h^{2}: \mathcal{D}^{b_{2}} B_{2} \Rightarrow$

Proof For brevity, we will omit writing that $\operatorname{Diff}\left(b_{1}, b_{2}\right)$, as well as applications of WD and $\operatorname{Ref}_{\sim i}^{i}$.

(1) $m / h^{0}: \mathcal{S} B_{1} \Rightarrow m / h^{1}: \mathcal{S} B_{1}, \ldots$

(2) same as the upper left sequent of R \&

$$
\begin{aligned}
& \frac{h^{0} \sim_{m}^{a} h, h^{0} \sim_{m}^{b_{1}} h^{1}, \ldots, m / h^{1}: \square^{b_{1}} B_{1}, \ldots \Rightarrow m / h^{1}: \mathcal{S} B_{1}, \ldots, m / h^{0}: \square^{b_{1}} B_{1} \quad(\mathbf{1})}{h^{0} \sim_{m}^{a} h, h^{0} \sim_{m}^{b_{1}} h^{1}, \ldots, m / h^{1}: \square^{b_{1}} B_{1}, \ldots \Rightarrow m / h^{1}: \mathcal{S} B_{1}, \ldots, m / h^{0}: \mathcal{D}^{b_{1}} B_{1}} R \mathcal{D}^{b_{1}} \\
& h^{0} \sim^{a} h, h^{0} \sim_{m}^{b_{1}} h^{1} \ldots m / h^{1} \cdot \square^{b_{1}} B_{1}, \ldots \Rightarrow m / h^{1}: S B_{1}, \ldots m / h^{0} \cdot \mathcal{D}^{b_{1}} B_{1} \& \mathcal{D}^{b_{2}} B_{2} \quad \text { (2) } R \& \\
& h^{0} \sim_{m}^{a} h, h^{0} \sim_{m_{1}}^{b_{1}} h^{1}, \ldots, h^{0}: \neg\left(\mathcal{D}^{b_{1}} B_{1} \& \mathcal{D}^{b_{2}} B_{2}\right), m / h^{1}: D^{b_{1}} B_{1}, \ldots \rightarrow m / h^{1}: \mathcal{S} B_{1}, \ldots \\
& h^{0} \sim_{m}^{a} h, h^{0} \sim_{m}^{b_{1}} h^{1}, \ldots, m / h^{0}: A \& \neg\left(\mathcal{D}^{b_{1}} B_{1} \& \mathcal{D}^{b_{2}} B_{2}\right), m / h^{1}, \square^{b_{1}} B_{1}, \ldots \Rightarrow m / h^{1}: S B_{1}, \ldots \& \\
& \frac{m}{h^{0} \sim_{m}^{a} h, h^{0} \sim_{m}^{b_{1}} h^{1}, \ldots, m / h: \square^{a}\left(A \& \neg\left(\mathcal{D}^{b_{1}} B_{1} \& \mathcal{D}^{b_{2}} B_{2}\right)\right), m / h^{1}: \square^{b_{1}} B_{1}, \ldots \Rightarrow m / h^{1}: \mathcal{S} B_{1}, \ldots} L \square^{a} \\
& h^{0} \sim a h^{1}, h^{0} \sim^{b_{1}} h^{1}, \ldots, a=b_{1}, h \sim^{a} h^{1}, m / h: \square^{a}\left(A \& \neg\left(\mathcal{D}^{b_{1}} B_{1} \& \mathcal{D}^{b_{2}} B_{2}\right)\right), m / h^{1}: \square^{b_{1}} B_{1}, \ldots \Rightarrow m / h^{1}: \mathcal{S} B_{1}, \ldots \text { Trans } \\
& \frac{\sim_{m} \sim_{m} h_{m}{ }_{m}^{b_{1}} h^{1}, \ldots, a=b_{1}, h \sim_{m}^{a} h^{1}, m / h: \square^{a}\left(A \& \neg\left(\mathcal{D}^{b_{1}} B_{1} \& \mathcal{D}^{b_{2}} B_{2}\right)\right), m / h^{1}: \square^{b_{1}} B_{1}, \ldots \Rightarrow m / h^{1}: \mathcal{S} B_{1}, \ldots}{h^{0} \sim_{m}} \operatorname{Repl}
\end{aligned}
$$

Lemma 4.3 Diff $\left(a, b_{1}, b_{2}\right), h^{2} \sim_{m}^{a} h, h^{1} \sim_{m}^{a} h, m / h: \mathcal{D}^{a}\left(A \& \neg\left(\mathcal{D}^{b_{1}} B_{1} \& \mathcal{D}^{b_{2}} B_{2}\right)\right), m / h^{1}$ : $\mathcal{D}^{b_{1}} B_{1}, m / h^{2}: \mathcal{D}^{b_{2}} B_{2} \Rightarrow$.

Proof For brevity we will omit writing $\operatorname{Diff}\left(a, b_{1}, b_{2}\right)$, as well as applications of WD and $\operatorname{Ref}_{\sim i}$.

(1) $m / h^{0}: \mathcal{S} B_{1} \Rightarrow m / h^{1}: \mathcal{S} B_{1}, \ldots$

(2) same as the upper left sequent of $R \&$

$$
\begin{aligned}
& \frac{h^{0} \sim_{m}^{a} h, h^{0} \sim_{m}^{b_{1}} h^{1}, h^{0} \sim_{m}^{b_{2}} h^{2}, \ldots, m / h^{1}: \square^{b_{1}} B_{1}, m / h^{2}: \square^{b_{2}} B_{2} \Rightarrow m / h^{1}: \mathcal{S} B_{1}, \ldots, m / h^{0}: \square^{b_{1}} B_{1} \quad(\mathbf{1})}{h^{0} \sim_{m}^{a} h, h^{0} \sim_{m}^{b_{1}} h^{1}, h^{0} \sim_{m}^{b_{2}} h^{2}, \ldots, m / h^{1}: \square^{b_{1}} B_{1}, m / h^{2}: \square^{b_{2}} B_{2} \Rightarrow m / h^{1}: \mathcal{S} B_{1}, \ldots, m / h^{0}: \mathcal{D}^{b_{1}} B_{1}} R \\
& h^{0} \sim_{m}^{a} h, h^{0} \sim_{m}^{b_{1}} h^{1}, h^{0} \sim_{m}^{b_{2}} h^{2}, \ldots, m / h^{1}: \square^{b_{1}} B_{1}, m / h^{2}: \square^{b_{2}} B_{2} \Rightarrow m / h^{1}: \mathcal{S} B_{1}, m / h^{2}: \mathcal{S} B_{2}, \ldots, m / h^{0}: \mathcal{D}^{b_{1}} B_{1} \& \mathcal{D}^{b_{2}} B_{2} \quad R \& \\
& h^{0} \sim_{m}^{a} h, h^{0} \sim_{m}^{b_{1}} h^{1}, h^{0} \sim_{m}^{b_{2}} h^{2}, \ldots, m / h^{0}: \neg\left(\mathcal{D}^{b_{1}} B_{1} \& \mathcal{D}^{b_{2}} B_{2}\right), m / h^{1}: \square^{b_{1}} B_{1}, m / h^{2}: \square^{b_{2}} B_{2} \Rightarrow m / h^{1}: \mathcal{S} B_{1}, m / h^{2}: \mathcal{S} B_{2}, \ldots L \neg \\
& h^{0} \sim_{m}^{a} h, h^{0} \sim_{m}^{b_{1}} h^{1}, h^{0} \sim_{m}^{b_{2}} h^{2}, \ldots, m / h^{0}: A \& \neg\left(\mathcal{D}^{b_{1}} B_{1} \& \mathcal{D}^{b_{2}} B_{2}\right), m / h^{1}: \square^{b_{1}} B_{1}, m / h^{2}: \square^{b_{2}} B_{2} \Rightarrow m / h^{1}: \mathcal{S} B_{1}, \ldots \\
& h^{0} \sim_{m}^{a} h, h^{0} \sim_{m}^{b_{1}} h^{1}, h^{0} \sim_{m}^{b_{2}} h^{2}, \ldots, m / h: \square^{a}\left(A \& \neg\left(\mathcal{D}^{b_{1}} B_{1} \& \mathcal{D}^{b_{2}} B_{2}\right)\right), m / h^{1}: \square^{b_{1}} B_{1}, m / h^{2}: \square^{b_{2}} B_{2} \Rightarrow m / h^{1}: \mathcal{S} B_{1}, \ldots \quad L \square^{a}
\end{aligned}
$$

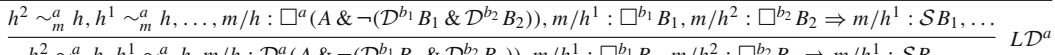

$$
\begin{aligned}
& h^{2} \sim_{m}^{a} h, h^{1} \sim_{m}^{a} h, m / h: \mathcal{D}^{a}\left(A \& \neg\left(\mathcal{D}^{b_{1}} B_{1} \& \mathcal{D}^{b_{2}} B_{2}\right)\right), m / h^{1}: \square^{b_{1}} B_{1}, m / h^{2}: \square^{b_{2}} B_{2} \Rightarrow m / h^{1}: \mathcal{S} B_{1}, \ldots \\
& \frac{h^{2} \sim_{m}^{a} h, h^{1} \sim_{m}^{a} h, m / h: \mathcal{D}^{a}\left(A \& \neg\left(\mathcal{D}^{b_{1}} B_{1} \& \mathcal{D}^{b_{2}} B_{2}\right)\right), m / h^{1}: \square^{b_{1}} B_{1}, m / h^{2}: \mathcal{D}^{b_{2}} B_{2} \Rightarrow m / h^{1}: \mathcal{S} B_{1}}{h^{2} \sim_{m}^{a} h, h^{1} \sim_{m}^{a} h, m / h: \mathcal{D}^{a}\left(A \& \neg\left(\mathcal{D}^{b_{1}} B_{1} \& \mathcal{D}^{b_{2}} B_{2}\right)\right), m / h^{1}: \mathcal{D}^{b_{1}} B_{1}, m / h^{2}: \mathcal{D}^{b_{2}} B_{2} \Rightarrow} L \mathcal{D}^{b_{1}}
\end{aligned}
$$

We can now show

$\mathbf{A I A}_{2}$

$\mathcal{D}^{a} A \&\left(\neg \mathcal{D}^{a}\left(A \& \neg \mathcal{D}^{b_{1}} B_{1}\right) \& \neg \mathcal{D}^{a}\left(A \& \neg \mathcal{D}^{b_{2}} B_{2}\right)\right) \& \operatorname{Diff}\left(b_{1}, b_{2}\right) \supset$

$\neg \mathcal{D}^{a}\left(A \& \neg\left(\mathcal{D}^{b_{1}} B_{1} \& \mathcal{D}^{b_{2}} B_{2}\right)\right)$ 


$$
\frac{h_{1} \sim_{m}^{i} h_{2}, m \in h_{1}, \ldots, h_{k+1}, \Gamma \Rightarrow \Delta \quad \ldots \quad h_{k} \sim_{m}^{i} h_{k+1}, m \in h_{1}, \ldots, h_{k+1}, \Gamma \Rightarrow \Delta}{m \in h_{1}, \ldots, m \in h_{k+1}, \Gamma \Rightarrow \Delta} A p c_{k}
$$

Fig. $2 \mathrm{G}^{2} \mathrm{Ldm}_{k}$

Proof We again omit writing $\operatorname{Diff}\left(b_{1}, b_{2}\right)$ for brevity.

(1) Using Lemma 4.2 for the case where $a=b_{1}$ and again for the case where $a=b_{2}$, Lemma 4.3 and an instance of A9, we obtain $m / h: \mathcal{D}^{a} A, h^{2} \sim_{m}^{a} h, h^{1} \sim_{m}^{a} h, m / h: \mathcal{D}^{a}\left(A \& \neg\left(\mathcal{D}^{b_{1}} B_{1} \& \mathcal{D}^{b_{2}} B_{2}\right)\right), m / h^{1}: \mathcal{D}^{b_{1}} B_{1}, m / h^{2}: \mathcal{D}^{b_{2}} B_{2} \Rightarrow$

(2), (3) $h \sim_{m}^{a} h^{\prime}, m / h: \mathcal{D}^{a} A \Rightarrow m / h^{\prime}: A$

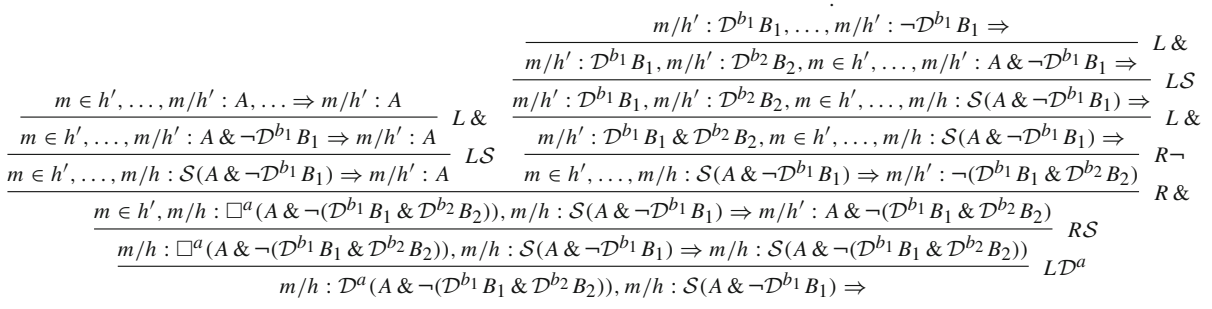

(5) The same as (4).

(1)

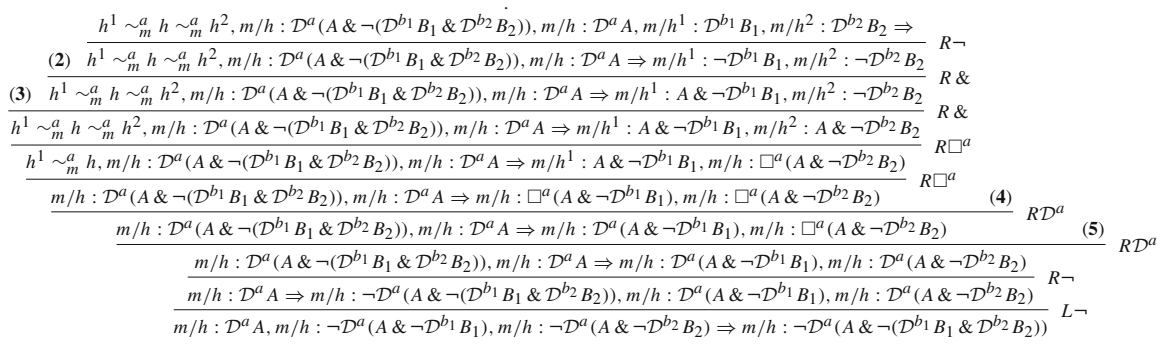

As already mentioned, the extension to the case of $n$ agents is long-winded, but straightforward.

\section{Logic $L_{k}$}

These logics are defined by the axiom $\mathrm{APC}_{k}$ for an appropriate $k$. To capture them, we now utilize logic $\mathrm{G} \mathrm{Ldm}_{k}$ (Fig. 2). As with independence of agents, we will demonstrate the proof of this axiom for the case of $k=2$, noting that the extension to $k$ is straightforward.

We begin with a lemma. Note that the Lemma* 5.1 refers to the result from Negri and Pavlović (2020).

Lemma $5.1 m / h^{1}: \mathcal{D}^{b} B_{1}, m / h^{1}: \neg B_{2}, m / h^{2}: \mathcal{D}^{b} B_{2} \Rightarrow m / h: \mathcal{D}^{b} B_{1}, m / h: \mathcal{D}^{b} B_{2}$. 
Proof (1)

\author{
Lemma* 5.1 \\ $h^{1} \sim_{m}^{b} h, m / h^{1}: \mathcal{D}^{b} B_{1}, m / h^{1}: \neg B_{2}, m / h^{2}: \mathcal{D}^{b} B_{2} \Rightarrow m / h: \mathcal{D}^{b} B_{1}, m / h: \mathcal{D}^{b} B_{2}$
}

(2) same as (1)

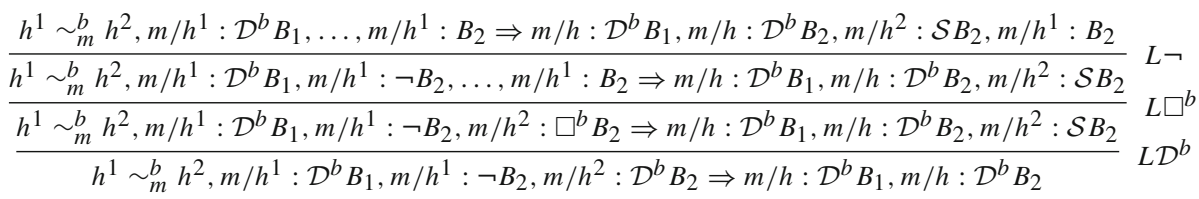

$\overline{m / h^{1}: \mathcal{D}^{b} B_{1}, m / h^{1}: \neg B_{2}, m / h^{2}: \mathcal{D}^{b} B_{2} \Rightarrow m / h: \mathcal{D}^{b} B_{1}, m / h: \mathcal{D}^{b} B_{2}} A P C_{2}$

We can now show

$\mathrm{APC}_{2}$

$\neg \mathcal{D}^{a}\left(A \& \neg\left(\mathcal{D}^{b} B_{1} \& \neg B_{2}\right)\right) \& \neg \mathcal{D}^{a}\left(A \& \neg \mathcal{D}^{b} B_{2}\right) \& \mathcal{D}^{a} A \& a \neq b \supset \mathcal{D}^{b} B_{1} \vee \mathcal{D}^{b} B_{2}$

\title{
Proof
}

(1)

$$
\frac{h^{1} \sim_{m}^{a} h, \ldots, m / h: \square^{a} A, m / h^{1}: A, \ldots \Rightarrow \ldots, m / h^{1}: A, \ldots, m / h: \mathcal{S} A}{\frac{h^{1} \sim_{m}^{a} h, \ldots, m / h: \square^{a} A, \ldots \Rightarrow \ldots, m / h^{1}: A, \ldots, m / h: \mathcal{S} A}{h^{1} \sim_{m}^{a} h, \ldots, m / h: \mathcal{D}^{a} A, \ldots \Rightarrow \ldots, m / h^{1}: A, \ldots} L D^{a}} \square^{a}
$$

(2) very similar to (1)

(3)

$$
\begin{aligned}
& \underline{m \in h^{\prime}, m / h^{\prime}: A, m / h^{\prime}: \neg \mathcal{D}^{b} B_{2}, m / h: \mathcal{S}\left(A \& \neg \mathcal{D}^{b} B_{2}\right), m / h: \square^{a} A, \ldots \Rightarrow \ldots, m / h^{\prime}: A} L \& \\
& m \in h^{\prime}, m / h^{\prime}: A \& \neg \mathcal{D}^{b} B_{2}, m / h: \mathcal{S}\left(A \& \neg \mathcal{D}^{b} B_{2}\right), m / h: \square^{a} A, \ldots \Rightarrow \ldots, m / h^{\prime}: A \\
& \underline{m \in h^{\prime}, m / h: \mathcal{S}\left(A \& \neg \mathcal{D}^{b} B_{2}\right), m / h: \square^{a} A, \ldots \Rightarrow \ldots, m / h^{\prime}: A} R \mathcal{S} \\
& \underline{m / h: \mathcal{S}\left(A \& \neg \mathcal{D}^{b} B_{2}\right), m / h: \square^{a} A, \ldots \Rightarrow \ldots, m / h: \mathcal{S} A} L \mathcal{D}^{a} \\
& m / h: \mathcal{S}\left(A \& \neg \mathcal{D}^{b} B_{2}\right), m / h: \mathcal{D}^{a} A, \ldots \Rightarrow \ldots
\end{aligned}
$$

(4) very similar to (3)

Lemma* 5.1

$m / h^{1}: \mathcal{D}^{b} B_{1}, m / h^{1}: \neg B_{2}, m / h^{2}: \mathcal{D}^{b} B_{2}, \ldots, m / h: \mathcal{D}^{a} A, m / h: a \neq b \Rightarrow m / h: \mathcal{D}^{b} B_{1}, m / h: \mathcal{D}^{b} B_{2}$

$m / h^{1}: \mathcal{D}^{b} B_{1} \& \neg B_{2}, m / h^{2}: \mathcal{D}^{b} B_{2}, \ldots, m / h: \mathcal{D}^{a} A, m / h: a \neq b \Rightarrow m / h: \mathcal{D}^{b} B_{1}, m / h: \mathcal{D}^{b} B_{2}$

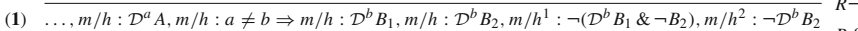

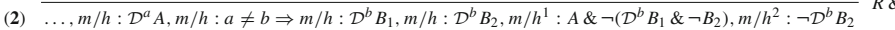

$h^{1} \sim_{m}^{a} h, h^{2} \sim_{m}^{a} h, m / h: \mathcal{D}^{a} A, m / h: a \neq b \Rightarrow m / h: \mathcal{D}^{b} B_{1}, m / h: \mathcal{D}^{b} B_{2}, m / h^{1}: A \& \neg\left(\mathcal{D}^{b} B_{1} \& \neg B_{2}\right), m / h^{2}: A \& \neg \mathcal{D}^{b} B_{2} \quad R \&$

$m / h: \mathcal{D}^{a} A, m / h: a \neq b \Rightarrow m / h: \mathcal{D}^{b} B_{1}, m / h: \mathcal{D}^{b} B_{2}, m / h: \square^{a}\left(A \& \neg\left(\mathcal{D}^{b} B_{1} \& \neg B_{2}\right)\right), m / h: \square^{a}\left(A \& \neg \mathcal{D}^{b} B_{2}\right)$

$m / h: \mathcal{D}^{a} A, m / h: a \neq b \Rightarrow m / h: \mathcal{D}^{b} B_{1}, m / h: \mathcal{D}^{b} B_{2}, m / h: \square^{a}\left(A \& \neg\left(\mathcal{D}^{b} B_{1} \& \neg B_{2}\right)\right), m / h: \mathcal{D}^{a}\left(A \& \neg \mathcal{D}^{b} B_{2}\right)$

(3) $R \mathcal{D}^{a}$

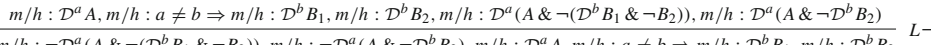

$m / h: \neg \mathcal{D}^{a}\left(A \& \neg\left(\mathcal{D}^{b} B_{1} \& \neg B_{2}\right)\right), m / h: \neg \mathcal{D}^{a}\left(A \& \neg \mathcal{D}^{b} B_{2}\right), m / h: \mathcal{D}^{a} A, m / h: a \neq b \Rightarrow m / h: \mathcal{D}^{b} B_{1}, m / h: \mathcal{D}^{b} B_{2}$ 


\section{Concluding Remarks}

The axiomatization of dstit offered in Xu (1998) can be seen as the logic of deliberative stit proper, as it contains only that modality. However, we have shown in this paper that the sequent calculus approach to it from Negri and Pavlović (2020), based on the BT + AC semantics and shown there to capture the axiomatization in Belnap et al. (2001), can also successfully encompass it.

As the height of the derivation in any G3 system depends on the weight of its endsequent, the longer axioms invariably lead to extended derivations, which has been very evident in this paper. So, compared against that common semantic backdrop, we have at the same time illustrated the benefit of utilizing multiple modalities, as the increase in the complexity of the language is offset by the gain in the brevity of proofs.

But first and foremost we have in this paper demonstrated that the system presented in Negri and Pavlović (2020) successfully and readily captures either approach to dstit.

Funding Open access funding provided by University of Helsinki including Helsinki University Central Hospital.

\section{Compliance with Ethical Standards}

Conflict of Interest The authors declare that they have no conflict of interest.

Open Access This article is licensed under a Creative Commons Attribution 4.0 International License, which permits use, sharing, adaptation, distribution and reproduction in any medium or format, as long as you give appropriate credit to the original author(s) and the source, provide a link to the Creative Commons licence, and indicate if changes were made. The images or other third party material in this article are included in the article's Creative Commons licence, unless indicated otherwise in a credit line to the material. If material is not included in the article's Creative Commons licence and your intended use is not permitted by statutory regulation or exceeds the permitted use, you will need to obtain permission directly from the copyright holder. To view a copy of this licence, visit http://creativecommons.org/licenses/by/4.0/.

\section{Appendix A: Axiom A6}

In this appendix we give a full proof for the axiom A6.

\section{Left to right:}

(1)

$\frac{h^{\prime \prime} \sim_{m}^{i} h^{\prime}, m / h^{\prime \prime}: A, m / h^{\prime \prime}: B \& \neg \mathcal{D}^{i} B, h^{\prime} \sim_{m}^{i} h, m / h^{\prime}: B, \ldots \Rightarrow m / h: \mathcal{S}\left(\neg \mathcal{D}^{i} A \&\left(B \& \neg \mathcal{D}^{i} B\right)\right), m / h^{\prime \prime}: A, m / h^{\prime}: \mathcal{D}^{i} B}{h^{\prime \prime} \sim_{m}^{i} h^{\prime}, m / h^{\prime \prime}: A \&\left(B \& \neg \mathcal{D}^{i} B\right), h^{\prime} \sim_{m}^{i} h, m / h^{\prime}: B, \ldots \Rightarrow m / h: \mathcal{S}\left(\neg \mathcal{D}^{i} A \&\left(B \& \neg \mathcal{D}^{i} B\right)\right), m / h^{\prime \prime}: A, m / h^{\prime}: \mathcal{D}^{i} B} \quad L \&$
$\frac{h^{\prime \prime} \sim_{m}^{i} h^{\prime}, m / h^{\prime}: \square^{i}\left(A \&\left(B \& \neg \mathcal{D}^{i} B\right)\right), h^{\prime} \sim_{m}^{i} h, m / h^{\prime}: B, \ldots \Rightarrow m / h: \mathcal{S}\left(\neg \mathcal{D}^{i} A \&\left(B \& \neg \mathcal{D}^{i} B\right)\right), m / h^{\prime \prime}: A, m / h^{\prime}: \mathcal{D}^{i} B}{L} \quad L \square \square^{i}$
$\frac{m / h^{\prime}: \square^{i}\left(A \&\left(B \& \neg \mathcal{D}^{i} B\right)\right), h^{\prime} \sim_{m}^{i} h, m / h^{\prime}: B, \ldots \Rightarrow m / h: \mathcal{S}\left(\neg \mathcal{D}^{i} A \&\left(B \& \neg \mathcal{D}^{i} B\right)\right), m / h^{\prime}: \square{ }^{i} A, m / h^{\prime}: \mathcal{D}^{i} B}{m / h^{\prime}: \mathcal{D}^{i}\left(A \&\left(B \& \neg \mathcal{D}^{i} B\right)\right), h^{\prime} \sim_{m}^{i} h, m / h^{\prime}: B, \ldots \Rightarrow m / h: \mathcal{S}\left(\neg \mathcal{D}^{i} A \&\left(B \& \neg \mathcal{D}^{i} B\right)\right), m / h^{\prime}: \square{ }^{i} A, m / h^{\prime}: \mathcal{D}^{i} B} L \mathcal{D}^{i}$

(2a) very similar to (1)

$$
\begin{aligned}
& \underline{m / h^{\prime \prime \prime}: A, m \in h^{\prime \prime \prime}, m / h^{\prime \prime}: \square^{i} A, m \in h^{\prime \prime}, m / h^{\prime}: \mathcal{S} B, m / h^{\prime}: \mathcal{S} A, m / h^{\prime}: \mathcal{D}^{i}\left(A \&\left(B \& \neg \mathcal{D}^{i} B\right)\right), h^{\prime} \sim_{m}^{i} h, m / h^{\prime}: B, \ldots \Rightarrow m / h^{\prime \prime \prime}: A} L \mathcal{S}
\end{aligned}
$$

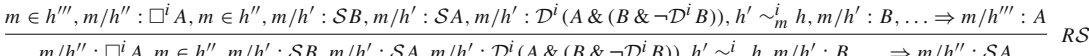

$$
\begin{aligned}
& \underline{m / h^{\prime \prime}: \square^{i} A, m \in h^{\prime \prime}, m / h^{\prime}: \mathcal{S} B, m / h^{\prime}: \mathcal{S} A, m / h^{\prime}: \mathcal{D}^{i}\left(A \&\left(B \& \neg \mathcal{D}^{i} B\right)\right), h^{\prime} \sim_{m}^{i} h, m / h^{\prime}: B, \ldots \Rightarrow m / h^{\prime \prime}: \mathcal{S} A} L \mathcal{D}^{i} \\
& \frac{m / h^{\prime \prime}: \mathcal{D}^{i} A, m \in h^{\prime \prime}, m / h^{\prime}: \mathcal{S} B, m / h^{\prime}: \mathcal{S} A, m / h^{\prime}: \mathcal{D}^{i}\left(A \&\left(B \& \neg \mathcal{D}^{i} B\right)\right), h^{\prime} \sim_{m}^{i} h, m / h^{\prime}: B, \ldots \Rightarrow}{m \in h^{\prime \prime}, m / h^{\prime}: \mathcal{S} B, m / h^{\prime}: \mathcal{S} A, m / h^{\prime}: \mathcal{D}^{i}\left(A \&\left(B \& \neg \mathcal{D}^{i} B\right)\right), h^{\prime} \sim_{m}^{i} h, m / h^{\prime}: B, \ldots \Rightarrow m / h^{\prime \prime}: \neg \mathcal{D}^{i} A} R \neg
\end{aligned}
$$




\section{(2c) very similar to $(2 b)$}

(2d)

$$
\begin{aligned}
& m / h^{\prime \prime}: B, m \in h^{\prime \prime}, m / h^{\prime}: \mathcal{S} B, m / h^{\prime}: \mathcal{S} A, m / h^{\prime}: \mathcal{D}^{i}\left(A \&\left(B \& \neg \mathcal{D}^{i} B\right)\right), h^{\prime} \sim_{m}^{i} h, m / h^{\prime}: B, \ldots \Rightarrow m / h^{\prime \prime}: B \\
& \frac{m \in h^{\prime \prime}, m / h^{\prime}: \mathcal{S} B, m / h^{\prime}: \mathcal{S} A, m / h^{\prime}: \mathcal{D}^{i}\left(A \&\left(B \& \neg \mathcal{D}^{i} B\right)\right), h^{\prime} \sim_{m}^{i} h, m / h^{\prime}: B, \ldots \Rightarrow m / h^{\prime \prime}: B \quad L \mathcal{S} \quad(2 c)}{m \in h^{\prime \prime}, m / h^{\prime}: \mathcal{S} B, m / h^{\prime}: \mathcal{S} A, m / h^{\prime} \cdot \mathcal{D}^{i}\left(A \&\left(B \& \neg \mathcal{D}^{i} B\right)\right), h^{\prime} \sim^{i} h, m / h^{\prime}: B, \ldots m / h^{\prime \prime}: B \& \neg \mathcal{D}^{i} B} R \&
\end{aligned}
$$

(2)

(2b)

(2d)

$\frac{(2 a) \quad m / h^{\prime}: \mathcal{S} B, m / h^{\prime}: \mathcal{S} A, m / h^{\prime}: \mathcal{D}^{i}\left(A \&\left(B \& \neg \mathcal{D}^{i} B\right)\right), h^{\prime} \sim_{m}^{i} h, m / h^{\prime}: B, \ldots \Rightarrow m / h: \mathcal{S}\left(\neg \mathcal{D}^{i} A \&\left(B \& \neg \mathcal{D}^{i} B\right)\right)}{m / h^{\prime}: \mathcal{S} A, m / h^{\prime}: \mathcal{D}^{i}\left(A \&\left(B \& \neg \mathcal{D}^{i} B\right)\right), h^{\prime} \sim_{m}^{i} h, m / h^{\prime}: B, \ldots \Rightarrow m / h: \mathcal{S}\left(\neg \mathcal{D}^{i} A \&\left(B \& \neg \mathcal{D}^{i} B\right)\right), m / h^{\prime}: \mathcal{D}^{i} B} R \mathcal{D}^{i}$

(1)

(2)

$\frac{m / h^{\prime}: \mathcal{D}^{i}\left(A \&\left(B \& \neg \mathcal{D}^{i} B\right)\right), h^{\prime} \sim{ }_{m}^{i} h, m / h^{\prime}: B, \ldots \Rightarrow m / h: \mathcal{S}\left(\neg \mathcal{D}^{i} A \&\left(B \& \neg \mathcal{D}^{i} B\right)\right), m / h^{\prime}: \mathcal{D}^{i} A, m / h^{\prime}: \mathcal{D}^{i} B}{R \mathcal{D}^{i}}$
$\frac{h^{\prime} \sim_{m}^{i} h, m / h^{\prime}: \neg \mathcal{D}^{i} A, m / h^{\prime}: B, m / h^{\prime}: \neg \mathcal{D}^{i} B, \ldots \Rightarrow m / h^{\prime}: \neg \mathcal{D}^{i}\left(A \&\left(B \& \neg \mathcal{D}^{i} B\right)\right), m / h: \mathcal{S}\left(\neg \mathcal{D}^{i} A \&\left(B \& \neg \mathcal{D}^{i} B\right)\right)}{L} L, R \neg$
$\frac{h^{\prime} \sim_{m}^{i} h, m / h^{\prime}: \neg \mathcal{D}^{i} A \&\left(B \& \neg \mathcal{D}^{i} B\right), \ldots \Rightarrow m / h^{\prime}: \neg \mathcal{D}^{i}\left(A \&\left(B \& \neg \mathcal{D}^{i} B\right)\right), m / h: \mathcal{S}\left(\neg \mathcal{D}^{i} A \&\left(B \& \neg \mathcal{D}^{i} B\right)\right)}{h^{\prime} \sim_{m}^{i} h, m / h: \square^{i}\left(\neg \mathcal{D}^{i} A \&\left(B \& \neg \mathcal{D}^{i} B\right)\right) \Rightarrow m / h^{\prime}: \neg \mathcal{D}^{i}\left(A \&\left(B \& \neg \mathcal{D}^{i} B\right)\right), m / h: \mathcal{S}\left(\neg \mathcal{D}^{i} A \&\left(B \& \neg \mathcal{D}^{i} B\right)\right)}$
$\frac{m / h: \square}{} \square^{i}\left(\neg \mathcal{D}^{i} A \&\left(B \& \neg \mathcal{D}^{i} B\right)\right) \Rightarrow m / h: \square^{i} \neg \mathcal{D}^{i}\left(A \&\left(B \& \neg \mathcal{D}^{i} B\right)\right), m / h: \mathcal{S}\left(\neg \mathcal{D}^{i} A \&\left(B \& \neg \mathcal{D}^{i} B\right)\right)$

$$
\frac{m \in h^{\prime}, m / h^{\prime \prime \prime \prime}: \mathcal{S}\left(A \&\left(B \& \neg \mathcal{D}^{i} B\right)\right), m / h^{\prime}: A, m / h^{\prime}:\left(B \& \neg \mathcal{D}^{i} B\right), \ldots \Rightarrow m / h^{\prime}: A, \ldots}{\frac{m \in h^{\prime}, m / h^{\prime \prime \prime \prime}: \mathcal{S}\left(A \&\left(B \& \neg \mathcal{D}^{i} B\right)\right), m / h^{\prime}: A \&\left(B \& \neg \mathcal{D}^{i} B\right), \ldots \Rightarrow m / h^{\prime}: A, \ldots}{\frac{m \in h^{\prime}, m / h^{\prime \prime \prime \prime}: \mathcal{S}\left(A \&\left(B \& \neg \mathcal{D}^{i} B\right)\right), \ldots \Rightarrow m / h^{\prime}: A, \ldots}{m / h^{\prime \prime \prime \prime}: \mathcal{S}\left(A \&\left(B \& \neg \mathcal{D}^{i} B\right)\right), \ldots \Rightarrow m / h^{\prime}: A, \ldots}} L \mathcal{S}} L
$$

(4b)

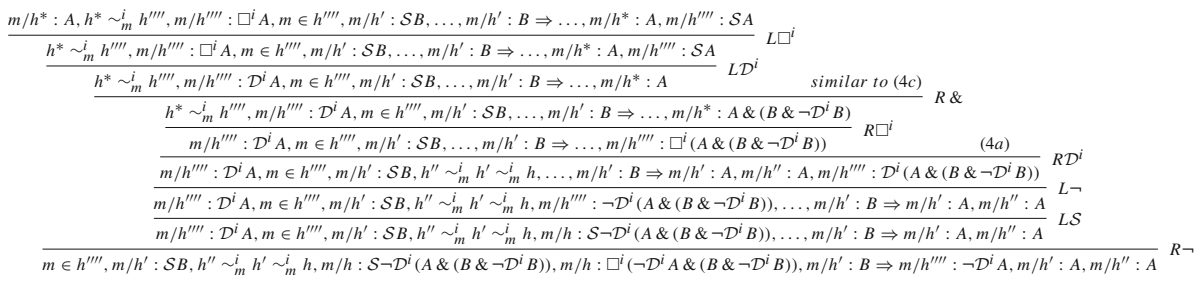

(4c)

$\frac{m \in h^{\prime \prime \prime \prime}, m / h^{\prime}: \mathcal{S} B, m / h^{\prime \prime \prime \prime}: B, \ldots \Rightarrow m / h^{\prime \prime \prime \prime}: B, \ldots}{m \in h^{\prime \prime \prime \prime}, m / h^{\prime}: \mathcal{S} B, \ldots \Rightarrow m / h^{\prime \prime \prime \prime}: B, \ldots} L \mathcal{S}$

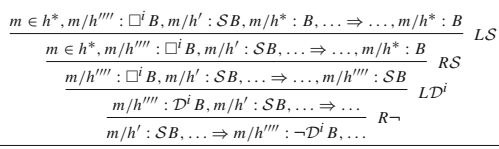

(4b) $m \in h^{\prime \prime \prime \prime}, m / h^{\prime}: \mathcal{S} B, h^{\prime \prime} \sim_{m}^{i} h^{\prime} \sim_{m}^{i} h, m / h: \mathcal{S} \neg \mathcal{D}^{i}\left(A \&\left(B \& \neg \mathcal{D}^{i} B\right)\right), m / h: \square^{i}\left(\neg \mathcal{D}^{i} A \&\left(B \& \neg \mathcal{D}^{i} B\right)\right), m / h^{\prime}: B \Rightarrow m / h^{\prime \prime \prime \prime}: B \& \neg \mathcal{D}^{i} B, m / h^{\prime}: A, m / h^{\prime \prime}: A \quad R \&$ $\frac{(4 b) m \in h^{\prime \prime}, m / h}{m \in h^{\prime \prime \prime \prime}, m / h^{\prime}: \mathcal{S} B, h^{\prime \prime} \sim_{m}^{i} h^{\prime} \sim_{m}^{i} h, m / h: \mathcal{S} \neg \mathcal{D}^{i}\left(A \&\left(B \& \neg \mathcal{D}^{i} B\right)\right), m / h: \square^{i}\left(\neg \mathcal{D}^{i} A \&\left(B \& \neg \mathcal{D}^{i} B\right)\right), m / h^{\prime}: B \Rightarrow m / h^{\prime \prime \prime \prime}: \neg \mathcal{D}^{i} A \&\left(B \& \neg \mathcal{D}^{i} B\right), m / h^{\prime}: A, m / h^{\prime \prime}: A} R \&$

$m / h^{\prime}: \mathcal{S} B, h^{\prime \prime} \sim_{m}^{i} h^{\prime} \sim_{m}^{i} h, m / h: \mathcal{S} \neg \mathcal{D}^{i}\left(A \&\left(B \& \neg \mathcal{D}^{i} B\right)\right), m / h: \square{ }^{i}\left(\neg \mathcal{D}^{i} A \&\left(B \& \neg \mathcal{D}^{i} B\right)\right), m / h^{\prime}: B \Rightarrow m / h: \mathcal{S}\left(\neg \mathcal{D}^{i} A \&\left(B \& \neg \mathcal{D}^{i} B\right)\right), m / h^{\prime}: A, m / h^{\prime \prime}: A$ 
(4d)

$$
\frac{h^{\prime \prime \prime} \sim_{m}^{i} h^{\prime \prime} \sim_{m}^{i} h^{\prime} \sim_{m}^{i} h, \ldots, m / h: \square^{i}\left(\neg \mathcal{D}^{i} A \&\left(B \& \neg \mathcal{D}^{i} B\right)\right), m / h^{\prime \prime \prime}: \neg \mathcal{D}^{i} A, m / h^{\prime \prime \prime}: B, m / h^{\prime \prime \prime}: \neg \mathcal{D}^{i} B, \ldots \Rightarrow \ldots, m / h^{\prime \prime \prime}: B}{\frac{h^{\prime \prime \prime} \sim_{m}^{i} h^{\prime \prime} \sim_{m}^{i} h^{\prime} \sim_{m}^{i} h, \ldots, m / h: \square^{i}\left(\neg \mathcal{D}^{i} A \&\left(B \& \neg \mathcal{D}^{i} B\right)\right), m / h^{\prime \prime \prime}: \neg \mathcal{D}^{i} A \&\left(B \& \neg \mathcal{D}^{i} B\right), \ldots \Rightarrow \ldots, m / h^{\prime \prime \prime}: B}{h^{\prime \prime \prime} \sim i} \sim^{i \prime} \sim i} L h^{i} \sim^{i} \sim^{i} h, L \&
$$
\begin{tabular}{c}
$\frac{h^{\prime \prime} \sim_{m}^{i} h^{\prime} \sim_{m}^{i} h, m / h: \mathcal{S} \neg \mathcal{D}^{i}\left(A \&\left(B \& \neg \mathcal{D}^{i} B\right)\right), m / h: \square^{i}\left(\neg \mathcal{D}^{i} A \&\left(B \& \neg \mathcal{D}^{i} B\right)\right), m / h^{\prime}: B \Rightarrow m / h: \mathcal{S}\left(\neg \mathcal{D}^{i} A \&\left(B \& \neg \mathcal{D}^{i} B\right)\right), m / h^{\prime}: A, m / h^{\prime \prime}: A, m / h^{\prime}: \square \square^{i} B}{R \square} \quad(4 c)$ \\
\hline$h^{\prime \prime} \sim_{m}^{i} h^{\prime} \sim_{m}^{i} h, m / h: \mathcal{S} \neg \mathcal{D}^{i}\left(A \&\left(B \& \neg \mathcal{D}^{i} B\right)\right), m / h: \square^{i}\left(\neg \mathcal{D}^{i} A \&\left(B \& \neg \mathcal{D}^{i} B\right)\right), m / h^{\prime}: B \Rightarrow m / h: \mathcal{S}\left(\neg \mathcal{D}^{i} A \&\left(B \& \neg \mathcal{D}^{i} B\right)\right), m / h^{\prime}: A, m / h^{\prime \prime}: A, m / h^{\prime}: \mathcal{D}^{i} B$
\end{tabular} $\frac{h^{\prime} \sim{ }_{m}^{i} h, m / h: \mathcal{S} \neg \mathcal{D}^{i}\left(A \&\left(B \& \neg \mathcal{D}^{i} B\right)\right), m / h: \square^{i}\left(\neg \mathcal{D}^{i} A \&\left(B \& \neg \mathcal{D}^{i} B\right)\right), m / h^{\prime}: B \Rightarrow m / h: \mathcal{S}\left(\neg \mathcal{D}^{i} A \&\left(B \& \neg \mathcal{D}^{i} B\right)\right), m / h^{\prime}: A, m / h^{\prime}: \square^{i} A, m / h^{\prime}: \mathcal{D}^{i} B}{R} \square^{i}$

$(4 \mathrm{e})$

$(4 d)$

$\frac{\ldots, m / h^{\prime}: \mathcal{S} A, m / h^{\prime}: A \Rightarrow \ldots, m / h^{\prime}: A, \ldots}{\ldots, m / h^{\prime}: \mathcal{S} A \Rightarrow \ldots, m / h^{\prime}: A, \ldots} L \mathcal{S}$

$h^{\prime} \sim_{m}^{i} h, m / h: \mathcal{S} \neg \mathcal{D}^{i}\left(A \&\left(B \& \neg \mathcal{D}^{i} B\right)\right), m / h: \square^{i}\left(\neg \mathcal{D}^{i} A \&\left(B \& \neg \mathcal{D}^{i} B\right)\right), m / h^{\prime}: B \Rightarrow m / h: \mathcal{S}\left(\neg \mathcal{D}^{i} A \&\left(B \& \neg \mathcal{D}^{i} B\right)\right), m / h^{\prime}: A, m / h^{\prime}: \mathcal{D}^{i} A, m / h^{\prime}: \mathcal{D}^{i} B$ $h^{\prime} \sim{ }_{m}^{i} h, m / h: \mathcal{S} \neg \mathcal{D}^{i}\left(A \&\left(B \& \neg \mathcal{D}^{i} B\right)\right), m / h: \square^{i}\left(\neg \mathcal{D}^{i} A \&\left(B \& \neg \mathcal{D}^{i} B\right)\right), m / h^{\prime}: \neg \mathcal{D}^{i} A, m / h^{\prime}: B, m / h^{\prime}: \neg \mathcal{D}^{i} B \Rightarrow m / h: \mathcal{S}\left(\neg \mathcal{D}^{i} A \&\left(B \& \neg \mathcal{D}^{i} B\right)\right), m / h^{\prime}: A \quad L \neg L$

$h^{\prime} \sim{ }_{m}^{i} h, m / h: \mathcal{S} \neg \mathcal{D}^{i}\left(A \&\left(B \& \neg \mathcal{D}^{i} B\right)\right), m / h: \square{ }^{i}\left(\neg \mathcal{D}^{i} A \&\left(B \& \neg \mathcal{D}^{i} B\right)\right), m / h^{\prime}: \neg \mathcal{D}^{i} A \&\left(B \& \neg \mathcal{D}^{i} B\right) \Rightarrow m / h: \mathcal{S}\left(\neg \mathcal{D}^{i} A \&\left(B \& \neg \mathcal{D}^{i} B\right)\right), m / h^{\prime}: A$

(4)

$(4 e)$

$m / h^{\prime}: B \& \neg \mathcal{D}^{i} B \Rightarrow m / h^{\prime}: B \& \neg \mathcal{D}^{i} B$

$\ldots, m / h^{\prime}: \neg \mathcal{D}^{i} A \&\left(B \& \neg \mathcal{D}^{i} B\right) \Rightarrow \ldots, m / h^{\prime}: B \& \neg \mathcal{D}^{i} B$

$h^{\prime} \sim_{m}^{i} h, m / h: \mathcal{S} \neg \mathcal{D}^{i}\left(A \&\left(B \& \neg \mathcal{D}^{i} B\right)\right), m / h: \square^{i}\left(\neg \mathcal{D}^{i} A \&\left(B \& \neg \mathcal{D}^{i} B\right)\right), m / h^{\prime}: \neg \mathcal{D}^{i} A \&\left(B \& \neg \mathcal{D}^{i} B\right) \Rightarrow m / h: \mathcal{S}\left(\neg \mathcal{D}^{i} A \&\left(B \& \neg \mathcal{D}^{i} B\right)\right), m / h^{\prime}: A \&\left(B \& \neg \mathcal{D}^{i} B\right) \quad R \&$

$\frac{h^{\prime} \sim_{m}^{i} h, m / h: \mathcal{S} \neg \mathcal{D}^{i}\left(A \&\left(B \& \neg \mathcal{D}^{i} B\right)\right), m / h: \square^{i}\left(\neg \mathcal{D}^{i} A \&\left(B \& \neg \mathcal{D}^{i} B\right)\right) \Rightarrow m / h: \mathcal{S}\left(\neg \mathcal{D}^{i} A \&\left(B \& \neg \mathcal{D}^{i} B\right)\right), m / h^{\prime}: A \&\left(B \& \neg \mathcal{D}^{i} B\right)}{m / h: \mathcal{S} \neg \mathcal{D}^{i}\left(A \&\left(B \& \neg \mathcal{D}^{i} B\right)\right), m / h: \square \square^{i}\left(\neg \mathcal{D}^{i} A \&\left(B \& \neg \mathcal{D}^{i} B\right)\right) \Rightarrow m / h: \mathcal{S}\left(\neg \mathcal{D}^{i} A \&\left(B \& \neg \mathcal{D}^{i} B\right)\right), m / h: \square \square^{i}\left(A \&\left(B \& \neg \mathcal{D}^{i} B\right)\right)}$

(5a)

$$
\begin{aligned}
& h \sim_{m}^{i} h^{\prime \prime}, \ldots, m / h: \mathcal{S}\left(A \&\left(B \& \neg \mathcal{D}^{i} B\right)\right), m / h^{\prime \prime}: A, m / h^{\prime \prime}:\left(B \& \neg \mathcal{D}^{i} B\right), \ldots \Rightarrow m / h^{\prime \prime}: A \\
& h \sim_{m}^{i} h^{\prime \prime}, \ldots, m / h: \mathcal{S}\left(A \&\left(B \& \neg \mathcal{D}^{i} B\right)\right), m / h^{\prime \prime}: A \&\left(B \& \neg \mathcal{D}^{i} B\right), \ldots \Rightarrow m / h^{\prime \prime}: A \\
& h \sim_{m}^{i} h^{\prime \prime}, \ldots, m / h: \mathcal{S}\left(A \&\left(B \& \neg \mathcal{D}^{i} B\right)\right), \ldots \Rightarrow m / h^{\prime \prime}: A \\
& \ldots, m / h: \mathcal{S}\left(A \&\left(B \& \neg \mathcal{D}^{i} B\right)\right), \ldots \Rightarrow m / h: \square^{i} A \quad R \square \square^{i}
\end{aligned}
$$

(5b)

$$
\frac{m / h^{\prime}: A, m / h^{\prime}: B \& \neg \mathcal{D}^{i} B, m \in h^{\prime}, m / h: \mathcal{S}\left(A \&\left(B \& \neg \mathcal{D}^{i} B\right)\right), \ldots \Rightarrow m / h^{\prime}: B \& \neg \mathcal{D}^{i} B}{\frac{m / h^{\prime}: A \&\left(B \& \neg \mathcal{D}^{i} B\right), m \in h^{\prime}, m / h: \mathcal{S}\left(A \&\left(B \& \neg \mathcal{D}^{i} B\right)\right), \ldots \Rightarrow m / h^{\prime}: B \& \neg \mathcal{D}^{i} B}{m \in h^{\prime}, m / h: \mathcal{S}\left(A \&\left(B \& \neg \mathcal{D}^{i} B\right)\right), \ldots \Rightarrow m / h^{\prime}: B \& \neg \mathcal{D}^{i} B}} L \mathcal{S}
$$

(5)

$$
\begin{aligned}
& \frac{\ldots, m / h^{\prime}: \square^{i} A, m / h: \mathcal{S} A, m / h^{\prime \prime}: A \Rightarrow \ldots, m / h^{\prime \prime}: A}{\ldots, m / h^{\prime}: \square^{i} A, m / h: \mathcal{S} A \Rightarrow \ldots, m / h^{\prime}: \mathcal{S} A} R \mathcal{S} \\
& \text { (5a) } \quad \ldots, m / h^{\prime}: \mathcal{D}^{i} A, m / h: \mathcal{S} A \Rightarrow \ldots \mathcal{D}^{i} \\
& \overline{h \sim^{i} h, m / h^{\prime}: \mathcal{D}^{i} A, m \in h^{\prime}, m / h: \mathcal{S}\left(A \&\left(B \& \neg \mathcal{D}^{i} B\right)\right), \ldots \Rightarrow m / h: \mathcal{D}^{i} A} R \mathcal{D}^{i} \\
& \overline{\left.h \sim{ }_{m}^{i} h, m / h^{\prime}: \mathcal{D}^{i} A, m \in h^{\prime}, m / h: \mathcal{S}\left(A \&\left(B \& \neg \mathcal{D}^{i} B\right)\right), \ldots, m / h: \neg \mathcal{D}^{i} A, m / h:\left(B \& \neg \mathcal{D}^{i} B\right)\right) \Rightarrow L \neg} \\
& \left.h \sim^{i} h, m / h^{\prime}: \mathcal{D}^{i} A, m \in h^{\prime}, m / h \cdot \mathcal{S}\left(A \&\left(B \& \neg \mathcal{D}^{i} B\right)\right), \ldots, m / h \cdot \neg \mathcal{D}^{i} A \&\left(B \& \neg \mathcal{D}^{i} B\right)\right) \Rightarrow L \& \\
& \frac{\left.h \sim_{m}^{i} h, m / h^{\prime}: \mathcal{D}^{i} A, m \in h^{\prime}, m / h: \mathcal{S}\left(A \&\left(B \& \neg \mathcal{D}^{i} B\right)\right), \ldots, m / h: \neg \mathcal{D}^{i} A \&\left(B \& \neg \mathcal{D}^{i} B\right)\right) \Rightarrow}{\underline{m / h^{\prime}: \mathcal{D}^{i} A, m \in h^{\prime}, m / h: \mathcal{S}\left(A \&\left(B \& \neg \mathcal{D}^{i} B\right)\right), \ldots, m / h: \square^{i}\left(\neg \mathcal{D}^{i} A \&\left(B \& \neg \mathcal{D}^{i} B\right)\right) \Rightarrow} \quad L \square^{i}, R e f l} \\
& m \in h^{\prime}, m / h: \mathcal{S}\left(A \&\left(B \& \neg \mathcal{D}^{i} B\right)\right), \ldots, m / h: \square^{i}\left(\neg \mathcal{D}^{i} A \&\left(B \& \neg \mathcal{D}^{i} B\right)\right) \Rightarrow m / h^{\prime}: \neg \mathcal{D}^{i} A \\
& m \in h^{\prime}, m / h: \mathcal{S}\left(A \&\left(B \& \neg \mathcal{D}^{i} B\right)\right), \ldots, m / h: \square^{i}\left(\neg \mathcal{D}^{i} A \&\left(B \& \neg \mathcal{D}^{i} B\right)\right) \Rightarrow m / h^{\prime}: \neg \mathcal{D}^{i} A \&\left(B \& \neg \mathcal{D}^{i} B\right) \\
& m / h: \mathcal{S}\left(A \&\left(B \& \neg \mathcal{D}^{i} B\right)\right), \ldots, m / h: \square^{i}\left(\neg \mathcal{D}^{i} A \&\left(B \& \neg \mathcal{D}^{i} B\right)\right) \Rightarrow m / h: \mathcal{S}\left(\neg \mathcal{D}^{i} A \&\left(B \& \neg \mathcal{D}^{i} B\right)\right)
\end{aligned}
$$

(6)

(4)

(5)

$\ldots, m / h: \square^{i}\left(\neg \mathcal{D}^{i} A \&\left(B \& \neg \mathcal{D}^{i} B\right)\right) \Rightarrow m / h: \mathcal{S}\left(\neg \mathcal{D}^{i} A \&\left(B \& \neg \mathcal{D}^{i} B\right)\right), m / h: \mathcal{D}^{i}\left(A \&\left(B \& \neg \mathcal{D}^{i} B\right)\right)$ $m / h: \neg \mathcal{D}^{i}\left(A \&\left(B \& \neg \mathcal{D}^{i} B\right)\right), \ldots, m / h: \square^{i}\left(\neg \mathcal{D}^{i} A \&\left(B \& \neg \mathcal{D}^{i} B\right)\right) \Rightarrow m / h: \mathcal{S}\left(\neg \mathcal{D}^{i} A \&\left(B \& \neg \mathcal{D}^{i} B\right)\right)$ $m / h: \mathcal{S} \neg \mathcal{D}^{i}\left(A \&\left(B \& \neg \mathcal{D}^{i} B\right)\right), m / h: \square^{i}\left(\neg \mathcal{D}^{i} A \&\left(B \& \neg \mathcal{D}^{i} B\right)\right) \Rightarrow m / h: \mathcal{S}\left(\neg \mathcal{D}^{i} A \&\left(B \& \neg \mathcal{D}^{i} B\right)\right)$ 
(3)

(6)

$\frac{m / h: \square^{i}\left(\neg \mathcal{D}^{i} A \&\left(B \& \neg \mathcal{D}^{i} B\right)\right) \Rightarrow m / h: \mathcal{D}^{i} \neg \mathcal{D}^{i}\left(A \&\left(B \& \neg \mathcal{D}^{i} B\right)\right), m / h: \mathcal{S}\left(\neg \mathcal{D}^{i} A \&\left(B \& \neg \mathcal{D}^{i} B\right)\right)}{m / h: \mathcal{D}^{i}\left(\neg \mathcal{D}^{i} A \&\left(B \& \neg \mathcal{D}^{i} B\right)\right) \Rightarrow m / h: \mathcal{D}^{i} \neg \mathcal{D}^{i}\left(A \&\left(B \& \neg \mathcal{D}^{i} B\right)\right)} L^{R} \mathcal{D}^{i}$

\section{Right to left:}

(1a)

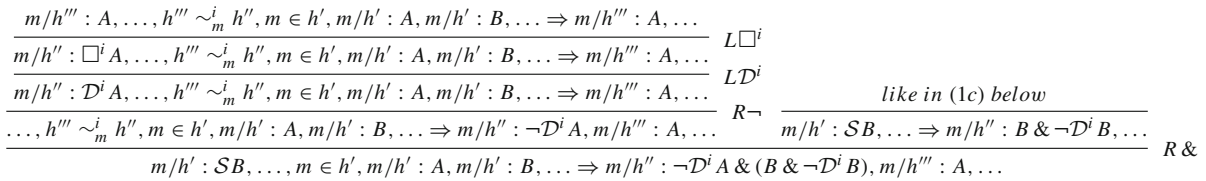

(1b)

$$
\frac{m / h^{\prime \prime \prime \prime}: A, m / h^{\prime \prime \prime \prime}: B, m / h^{\prime \prime \prime \prime}: \neg \mathcal{D}^{i} B, h^{\prime \prime \prime \prime} \sim_{m}^{i} h^{\prime}, \ldots \Rightarrow m / h^{\prime \prime}: \neg \mathcal{D}^{i} A \&\left(B \& \neg \mathcal{D}^{i} B\right), m / h^{\prime \prime \prime}: A \&\left(B \& \neg \mathcal{D}^{i} B\right), \ldots, m / h^{\prime \prime \prime \prime \prime}: B}{} \quad L \&
$$

(1c)

(1a) $\frac{m / h^{\prime}: \mathcal{S} B, \ldots \Rightarrow \ldots, m / h^{\prime \prime \prime}: B \quad m / h^{\prime}: \mathcal{S} B, \ldots \Rightarrow \ldots, m / h^{\prime \prime \prime}: \neg \mathcal{D}^{i} B}{m / h^{\prime}: \mathcal{S} B, \ldots, m \in h^{\prime}, m / h^{\prime}: A, m / h^{\prime}: B, \ldots \Rightarrow m / h^{\prime \prime}: \neg \mathcal{D}^{i} A \&\left(B \& \neg \mathcal{D}^{i} B\right), m / h^{\prime \prime \prime}: B \& \neg \mathcal{D}^{i} B, \ldots} R \&$

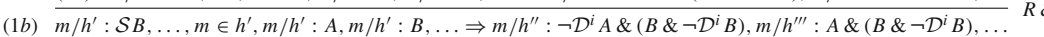
$\overline{h^{\prime \prime \prime} \sim_{m}^{i} h \sim_{m}^{i} h^{\prime \prime}, m \in h^{\prime}, m / h^{\prime}: A, m / h^{\prime}: B, \ldots \Rightarrow m / h^{\prime \prime}: \neg \mathcal{D}^{i} A \&\left(B \& \neg \mathcal{D}^{i} B\right), m / h^{\prime \prime \prime}: A \&\left(B \& \neg \mathcal{D}^{i} B\right), \ldots, m / h^{\prime}: \mathcal{D}^{i} B} R \mathcal{D}^{i}$ $h^{\prime \prime \prime} \sim_{m}^{i} h \sim_{m}^{i} h^{\prime \prime}, m \in h^{\prime}, m / h^{\prime}: A, m / h^{\prime}: B, m / h^{\prime}: \neg \mathcal{D}^{i} B, \ldots \Rightarrow m / h^{\prime \prime}: \neg \mathcal{D}^{i} A \&\left(B \& \neg \mathcal{D}^{i} B\right), m / h^{\prime \prime \prime}: A \&\left(B \& \neg \mathcal{D}^{i} B\right), \ldots$ $h^{\prime \prime \prime} \sim_{m}^{i} h \sim_{m}^{i} h^{\prime \prime}, m \in h^{\prime}, m / h^{\prime}: A \&\left(B \& \neg \mathcal{D}^{i} B\right), \ldots \Rightarrow m / h^{\prime \prime}: \neg \mathcal{D}^{i} A \&\left(B \& \neg \mathcal{D}^{i} B\right), m / h^{\prime \prime \prime}: A \&\left(B \& \neg \mathcal{D}^{i} B\right), \ldots$

$h^{\prime \prime \prime} \sim_{m}^{i} h \sim_{m}^{i} h^{\prime \prime}, m \in h^{\prime}, m / h^{\prime}: \mathcal{D}^{i}\left(A \&\left(B \& \neg \mathcal{D}^{i} B\right)\right) \Rightarrow m / h^{\prime \prime}: \neg \mathcal{D}^{i} A \&\left(B \& \neg \mathcal{D}^{i} B\right), m / h^{\prime \prime \prime}: A \&\left(B \& \neg \mathcal{D}^{i} B\right)$ $m \in h^{\prime}, m / h^{\prime}: \mathcal{D}^{i}\left(A \&\left(B \& \neg \mathcal{D}^{i} B\right)\right) \Rightarrow m / h: \square^{i}\left(\neg \mathcal{D}^{i} A \&\left(B \& \neg \mathcal{D}^{i} B\right)\right), m / h: \square^{i}\left(A \&\left(B \& \neg \mathcal{D}^{i} B\right)\right) \quad R \square^{i}, R \square^{i}$

(1d)

$$
\begin{aligned}
& \frac{\ldots, m / h^{\prime \prime}: \neg \mathcal{D}^{i} A, m / h^{\prime \prime}: B \& \neg \mathcal{D}^{i} B, m \in h^{\prime}, m / h^{\prime}: \square^{i}\left(A \&\left(B \& \neg \mathcal{D}^{i} B\right)\right) \Rightarrow m / h^{\prime \prime}: B \& \neg \mathcal{D}^{i} B, \ldots, m / h^{\prime}: \mathcal{D}^{i} A}{\ldots, m / h^{\prime \prime}: \neg \mathcal{D}^{i} A \&\left(B \& \neg \mathcal{D}^{i} B\right), m \in h^{\prime}, m / h^{\prime}: \square{ }^{i}\left(A \&\left(B \& \neg \mathcal{D}^{i} B\right)\right) \Rightarrow m / h^{\prime \prime}: B \& \neg \mathcal{D}^{i} B, \ldots, m / h^{\prime}: \mathcal{D}^{i} A} L \& \\
& \frac{\ldots, m / h: \mathcal{S}\left(\neg \mathcal{D}^{i} A \&\left(B \& \neg \mathcal{D}^{i} B\right)\right), m \in h^{\prime}, m / h^{\prime}: \square^{i}\left(A \&\left(B \& \neg \mathcal{D}^{i} B\right)\right) \Rightarrow m / h^{\prime \prime}: B \& \neg \mathcal{D}^{i} B, \ldots, m / h^{\prime}: \mathcal{D}^{i} A}{L}
\end{aligned}
$$

\section{Springer}




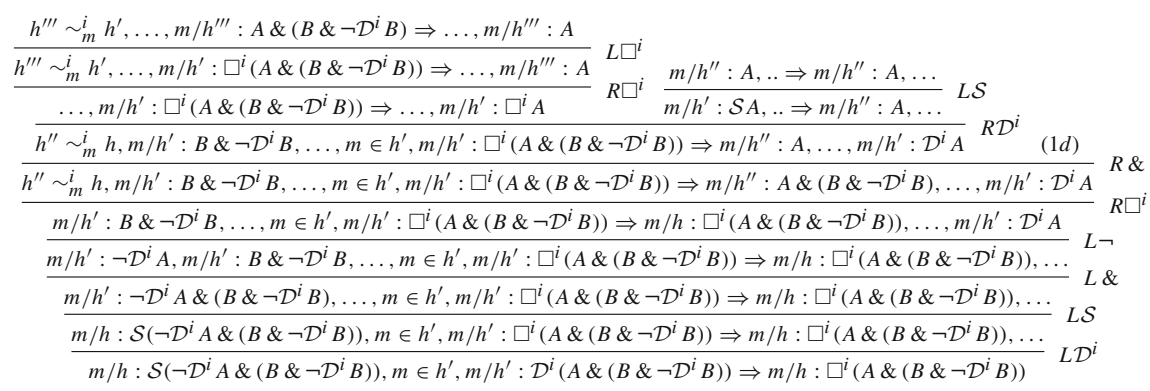

(1c)

$(1 e)$

$m \in h^{\prime}, m / h^{\prime}: \mathcal{D}^{i}\left(A \&\left(B \& \neg \mathcal{D}^{i} B\right)\right) \Rightarrow m / h: \mathcal{D}^{i}\left(\neg \mathcal{D}^{i} A \&\left(B \& \neg \mathcal{D}^{i} B\right)\right), m / h: \square{ }^{i}\left(A \&\left(B \& \neg \mathcal{D}^{i} B\right)\right) ~ R \mathcal{D}$

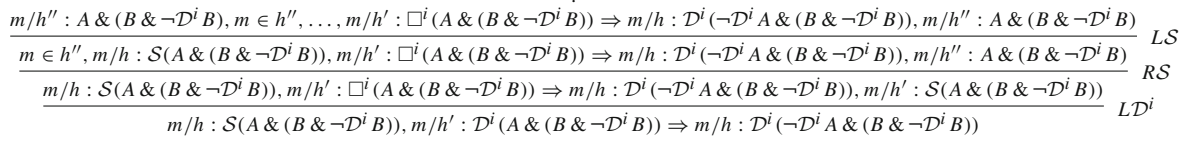

(1)

(2)

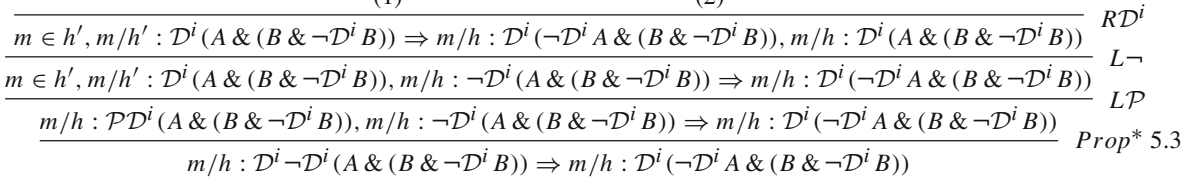

\section{References}

Balbiani, P., Herzig, A., \& Troquard, N. (2008). Alternative axiomatics and complexity of deliberative stit theories. Journal of Philosophical Logic, 37(4), 387-406.

Belnap, N., Perloff, M., \& Xu, M. (2001). Facing the future: Agents and choices in our indeterminist world. Oxford: Oxford University Press.

Chellas, B. (1980). Modal logic: An introduction. Cambridge: Cambridge University Press.

Lyon, T., \& van Berkel, K. (2019). Automating agential reasoning: Proof-calculi and syntactic decidability for stit logics. In International conference on principles and practice of multi-agent systems (pp 202-218). Berlin: Springer.

Negri, S. (2005). Proof analysis in modal logic. Journal of Philosophical Logic, 34(5-6), 507.

Negri, S., \& Orlandelli, E. (2019). Proof theory for quantified monotone modal logics. Logic Journal of the IGPL, 27(4), 478-506.

Negri, S., \& Pavlović, E. (2020). Proof-theoretic analysis of the logics of agency: The deliberative STIT. Studia Logica 1-35. https://doi.org/10.1007/s11225-020-09914-1.

Negri, S., \& Sbardolini, G. (2016). Proof analysis for Lewis counterfactuals. The Review of Symbolic Logic, 9(1), 44-75. 
Negri, S., \& von Plato, J. (1998). Cut elimination in the presence of axioms. Bulletin of Symbolic Logic, 4(4), $418-435$.

Negri, S., \& von Plato, J. (2001). Structural proof theory. Cambridge: Cambridge University Press.

Negri, S., \& von Plato, J. (2011). Proof analysis: A contribution to Hilbert's last problem. Cambridge: Cambridge University Press.

Olkhovikov, G., \& Wansing, H. (2018). An axiomatic system and a tableau calculus for stit imagination logic. Journal of Philosophical Logic, 47(2), 259-279.

Olkhovikov, G. K., \& Wansing, H. (2019). Simplified tableaux for stit imagination logic. Journal of Philosophical Logic, 48(6), 981-1001.

van Berkel, K., \& Lyon, T. (2019). Cut-free calculi and relational semantics for temporal stit logics. In: European Conference on Logics in Artificial Intelligence (pp. 803-819). Berlin: Springer

Wansing, H. (2006). Tableaux for multi-agent deliberative-stit logic. Advances in modal logic, 6, 503-520.

Wansing, H. (2017). Remarks on the logic of imagination. a step towards understanding doxastic control through imagination. Synthese, 194(8), 2843-2861.

Xu, M. (1998). Axioms for deliberative stit. Journal of Philosophical Logic, 27(5), 505-552.

Publisher's Note Springer Nature remains neutral with regard to jurisdictional claims in published maps and institutional affiliations.

Sara Negri is Professor of Mathematical Logic at the University of Genova. She has been Professor of Theoretical Philosophy at the University of Helsinki since 2015, as well as a Docent of Logic since 1998. After a $\mathrm{PhD}$ in Mathematics in 1996 at the University of Padova and research visits at the University of Amsterdam and Chalmers, she has been a Research Associate at the Imperial College in London, a Humboldt Fellow in Munich, and a visiting scientist at the Mittag-Leffler Institute in Stockholm and at the Hausdorff Institute for Mathematics in Bonn and a visiting professor at the Scuola Normale Superiore of Pisa and the University of Verona. Her research interests range from mathematical logic and philosophy of mathematics to proof theory and its applications to modal and non-classical logics.

Edi Pavlović was born in Rijeka, Croatia and studied for his bachelor's degree at the University of Rijeka. He continued his graduate studies at the Central European University, then in Budapest, where he earned his master's degree in 2012 and a Ph.D. in 2017, both in philosophy. After graduating he joined University of Helsinki in 2018 as a postdoctoral researcher on the project Modalities and conditionals: Systematic and historical studies with Prof. Negri the principal investigator. His research interests lie in philosophical logic, as well as proof theory and its applications to modal and non-classical logics in general, and particularly to logics of agency and free logics 